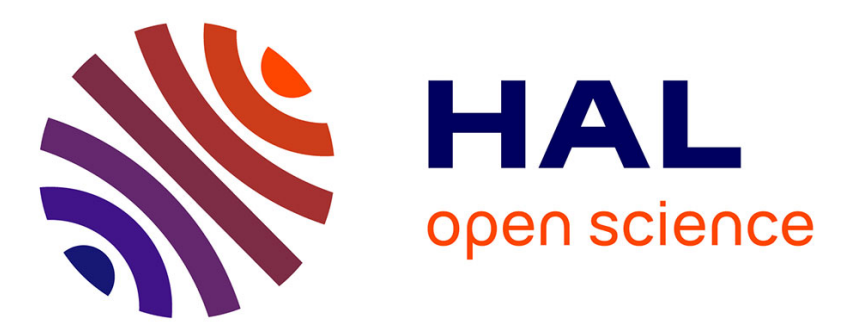

\title{
Adsorption, Desorption, and Crystallization of Aqueous Solutions in Nanopores
}

P. Jain, Olivier O. Vincent, A. D. Stroock

\section{To cite this version:}

P. Jain, Olivier O. Vincent, A. D. Stroock. Adsorption, Desorption, and Crystallization of Aqueous Solutions in Nanopores. Langmuir, 2019, 35, pp.3949-3962. 10.1021/acs.langmuir.8b04307 . hal02285864

\section{HAL Id: hal-02285864 \\ https://univ-lyon1.hal.science/hal-02285864}

Submitted on 12 May 2020

HAL is a multi-disciplinary open access archive for the deposit and dissemination of scientific research documents, whether they are published or not. The documents may come from teaching and research institutions in France or abroad, or from public or private research centers.
L'archive ouverte pluridisciplinaire HAL, est destinée au dépôt et à la diffusion de documents scientifiques de niveau recherche, publiés ou non, émanant des établissements d'enseignement et de recherche français ou étrangers, des laboratoires publics ou privés. 
Adsorption, desorption, and crystallization of aqueous solutions in nanopores

\author{
Piyush Jain, ${ }^{\dagger}$ Olivier Vincent, ${ }^{*,+, \S}$ and Abraham D. Stroock ${ }^{*,+,}$ \\ †Sibley School of Mechanical and Aerospace Engineering, Cornell University, Ithaca, New \\ York 14853, United States \\ $\ddagger$ Robert Frederick Smith School of Chemical and Biomolecular Engineering, Cornell \\ University, Ithaca, New York 14853, United States \\ \Kavli Institute at Cornell for Nanoscale Science, Cornell University, Ithaca, New York \\ 14853, United States \\ $\S$ Current address: University of Lyon, Université Claude Bernard Lyon 1, CNRS, Institut \\ Lumière Matière, F-69622, Villeurbanne, France \\ E-mail: olivier.vincent@univ-lyon1.fr; ads10@cornell.edu
}

\begin{abstract}
Probing nano-confined solutions in tortuous, mesoporous media is challenging due to pore size, complex pore connectivity, and coexistence of multiple components and phases. Here, we use optical reflectance to experimentally investigate the wetting and drying of a mesoporous medium with $\sim 3 \mathrm{~nm}$ diameter pores containing aqueous solutions of sodium chloride and lithium chloride. We show that the vapor activities, i.e., relative humidities, that correspond to optical features in the isotherms for solutions can be used to deduce the thermodynamic state of a nanoscopic solution that undergoes evaporation and crystallization upon drying, and condensation and deliquescence when increasing the relative humidity. We emphasize specific equilibrium states


of the system: the onset of draining during desorption and the end of filling during adsorption, percolation-induced scattering and crystallization. We find that theoretical arguments involving classical thermodynamics (modified Kelvin-Laplace Equation and classical nucleation theory) explain quantitatively the evolution of the optical features, and, thereby, the state of the solution, as a function of imposed vapor activity and solute concentration.

\section{Introduction}

Salt solutions frequently occupy the pore network of porous media in natural and technological contexts: Interaction of solutions with host matrices influence salt weathering due to stresses in the pore liquid within building materials; ${ }^{1,2}$ movement of contaminants in unsaturated soils lead to aquifer contamination; ${ }^{3} \mathrm{CO}_{2}$ is sequestered in brine by capillary trapping; ${ }^{4}$ and, nanoporous membranes are used for vapor phase desalination. ${ }^{5}$ Wetting and drying cycles of salt solutions in porous networks regulate critical aspects in each of these contexts. The localization of crystallization during drying govern different modes of damage in materials: crystal growth upon evaporation beneath the surface results in damage due to subflorescence, while crystallization on surface result in efflorescence-induced damage. ${ }^{6,7}$ The transport of solutions in ground-water within the capillary fringe has been proposed to affect the solute distributions in soils; this mode of transport has implications in defining the geochemical and biological activity of groundwater systems. ${ }^{8,9}$ However, insufficient understanding of thermodynamics of solution-filled porous networks hinders our ability to predict and engineer in these contexts.

The influence of wetting and drying of porous media by a multicomponent and multiphase fluids has been subject of various numerical ${ }^{10}$ and theoretical investigations. ${ }^{11,12}$ Advances in spatiotemporal studies of water and ion transport using 3D imaging techniques such as micro-CT scanning and Nuclear Magnetic Resonance(NMR) have helped the exploration of the local dynamics of solutions in macroporous materials. ${ }^{13,14}$ Isotherms of porous 

rials. ${ }^{15}$ Also, wetting and drying of mesoporous media presents rich variety of effects concerning hysteresis, dynamics and optical and acoustic signatures. ${ }^{16-18}$ Further, the shape of adsorption-desorption isotherms can be analyzed to provide insight into the dynamic and thermodynamic behavior of adsorbates under nanoconfinement and adsorbate-adsorbent interaction. ${ }^{16,19}$ Recently, our group used reflectance isotherms to study the dynamics and thermodynamics of pure liquid water in mesoporous material and the dynamics of spatiotemporal reflectance changes as function of imposed vapor pressure. ${ }^{20}$

In this paper, we use reflectance isotherms of porous samples filled with aqueous solutions of various concentration to explain, quantitatively, important characteristics of the phase behavior within the host (Figure $1(\mathrm{a}, \mathrm{b})$ ). We recorded changes in reflectance of mesoporous silicon layer filled with $\mathrm{LiCl}$ and $\mathrm{NaCl}$ solutions as function of step increases and decreases in vapor activity, $a$, referred to as the adsorption branch (Figure 1(b), filled squares) and the desorption branch (Figure 1(b), open circles), respectively. We refer to these curves as reflectance isotherms (Figure $1(\mathrm{~b})$ ). We define vapor activity, $a=p / p_{\text {sat }}$, where $p$ is the imposed vapor pressure and $p_{\text {sat }}$ is the saturation vapor pressure of pure water at the same temperature.

Figure 1(c-j) present sketches of states of the pore solution that we hypothesize are correlated with the the features of interest marked on reflectance isotherms: (i) solution completely fills the pores at the bulk-solution activity $\left(a_{\mathrm{s}}\right.$ - Figure $\left.1(\mathrm{c})\right)$, corresponding to the right-most point of the isotherms; On the desorption branch (decreasing $a$ ), (ii) curvature of solution-vapor interface increases with decreasing vapor activity $\left(a_{\mathrm{dt}}<a<a_{\mathrm{s}}\right.$ - Figure 1(d)) corresponding to the plateau at high activities; (iii) the meniscus reaches its maximum curvature, i.e., the tension (negative pressure) in the liquid reaches its largest magnitude and pore liquid starts to recede into the pores at the desorption-tension activity $\left(a_{\mathrm{dt}}-\right.$ Figure $1(\mathrm{e}$, f)), corresponding to initial rise in $\langle\Delta I / I\rangle$ in the desorption branch (open circles in Figure 1(b)) of the reflectance isotherm; (iv) crystallization in the pores occurs at the crystalliza- 
(a) $\operatorname{Dry}(a<<1) \quad \operatorname{Wet}(a \rightarrow 1)$

(c) $a=a_{\mathrm{s}}$
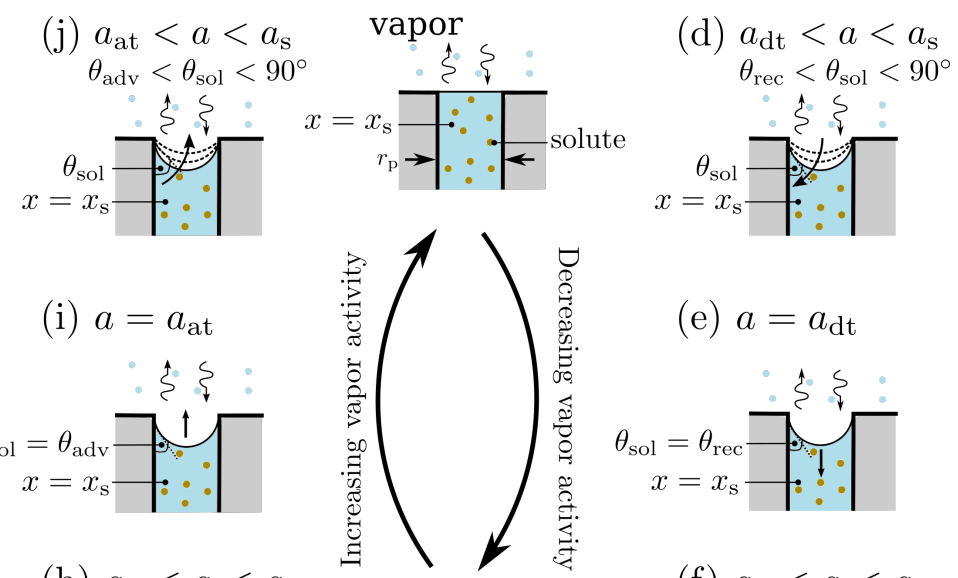

(e) $a=a_{\mathrm{dt}}$

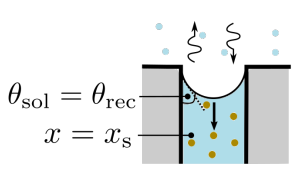

(f) $a_{\mathrm{c}}<a<a_{\mathrm{dt}}$

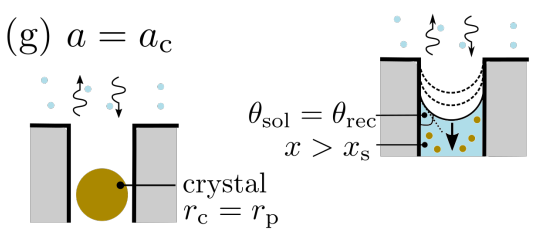

Figure 1: Optical reflectance isotherms of nanoporous medium filled with an aqueous solution. (a) Optical images (top) show top-view of the dry and wet states of sample. Side-view sketch of the sample (bottom). (b) Typical reflectance isotherm (here with $\mathrm{NaCl}$ as solute). (c-j) Theoretical sketches of the states of the pore solution for features noted on the isotherms in (b). The sample is entirely filled with solution at activity, $a=a_{\mathrm{s}}$, corresponding to a mole fraction of solute, $x_{\mathrm{s}}$; the solution-vapor meniscus is flat (c). Upon lowering activity, capillary pressure increases as the liquid-vapor meniscus curves (d) until $\theta_{\text {sol }}=\theta_{\text {rec }}$ as the vapor reaches desorption-tension activity, $a_{\mathrm{dt}}(\mathrm{e})$. Upon further lowering activity, desorption starts with receding menisci; the vapor pressure of receding solution decreases as the concentration of dissolved solute increases, $x>x_{\mathrm{s}}$ (f), until the vapor reaches the crystallization activity, $a_{\mathrm{c}}$, where supersaturation is sufficient for the critical radius of crystal nucleus, $r_{\mathrm{c}}$, to be less than or equal to the pore radius, $r_{\mathrm{p}}(\mathrm{g})$. On adsorption, the crystal deliquesces into solution, then pore-solution re-invades with the liquid-vapor meniscus making a contact angle, $\theta_{\text {sol }}=\theta_{\text {adv }}$, with the pore wall $(\mathrm{g})$ until the pore solution reaches the mouth of the pores at adsorption-tension activity, $a_{\text {at }}(\mathrm{i})$. The vapor-liquid menisci relax $(\mathrm{j})$, then vapor reaches bulk-solution activity, $a_{\mathrm{s}}$, above which there is bulk condensation of solution on the external surface. On both adsorption and desorption branches, scattering reaches maxima at vapor activities referred to as adsorption-peak activity, $a_{\mathrm{ap}}$, and desorption-peak activity, $a_{\mathrm{dp}}$. 
tion activity $\left(a_{\mathrm{c}}\right.$ - Figure $1(\mathrm{~g})$ ), corresponding to the lower point of closure of the hysteresis loop; On the adsorption branch (increasing $a$ ), (v) the crystal deliquesces and the pore liquid advances to the mouth of the pores at the adsorption-tension activity $\left(a_{\text {at }}\right.$-Figure $1(\mathrm{~h}, \mathrm{i})$ ), corresponding to the upper closure point of the hysteresis loops; (vi) the curvature of the solution-vapor interface decreases with increasing vapor activity $\left(a_{\mathrm{at}}<a<a_{\mathrm{s}}\right.$ - Figure $\left.1(\mathrm{j})\right)$ corresponding to the plateau on adsorption branch, until the solution completely fills the pores at the bulk-solution activity, $a_{\mathrm{s}}$. As discussed in more detail in the following, we interpret the peaks in both branches as due to light scattering from heterogeneous distributions of gas-filled and liquid-filled pores. Using a single porous substrate (anodized, mesoporous silicon, pore size $\sim 3 \mathrm{~nm}$ ), we collected isotherms for pure water (Figure 2), aqueous solutions of Sodium Chloride ( $\mathrm{NaCl}$, Figure 3) and aqueous solutions of Lithium Chloride ( $\mathrm{LiCl}$, Figure 4).

In this study, we analyze reflectance isotherms as a function of amount of solute filling the pores to provide quantitative support for the hypotheses in Figures 1(c)-1(j). We organize the remainder of the paper as follows: we describe the experimental methods for measuring the reflectance isotherms, we present theoretical equations based on classical thermodynamics to explain the evolution of features with varying activity of solution (equivalently, concentration of solution), we report and compare experimental activities with the theoretical predictions in accordance with our hypotheses, and, finally, we discuss the implications of these results for our understanding of the thermodynamic state of pore solutions.

\section{Methods}

A mesoporous silicon layer of $5 \mu \mathrm{m}$-thickness was formed by anodization as described previously (Figure 1(a)). ${ }^{21}$ Briefly, we formed a $5 \mu \mathrm{m}$-thick layer of mesoporous silicon (PoSi) in the surface of a polished, p-type silicon (Si) wafer of $\langle 111\rangle$ crystal orientation and $1-10$ $\Omega-\mathrm{cm}$ resistivity, by anodization in a mixture of 1:1 $49 \%$ hydrofluoric acid/pure ethanol at a 
current density of $20 \mathrm{~mA} / \mathrm{cm}^{2}$ for $5 \mathrm{~min}$. We then thermally oxidized the substrate at $700{ }^{\circ} \mathrm{C}$ in pure oxygen for $30 \mathrm{~s}$ to increase hydrophilicity and stabilize the porous layer. We expect a laterally connected pore structure with porosity, $\phi=0.45$ and a typical pore radius, $r_{\mathrm{p}}$, of $1.4 \mathrm{~nm}$ (pore radius distribution is centered on $1.4 \mathrm{~nm}$ with a half-width-at-half-maximum of $0.4 \mathrm{~nm}$, as estimated in previous studies from nitrogen sorption porosimetry and dynamic flow methods). ${ }^{21}$

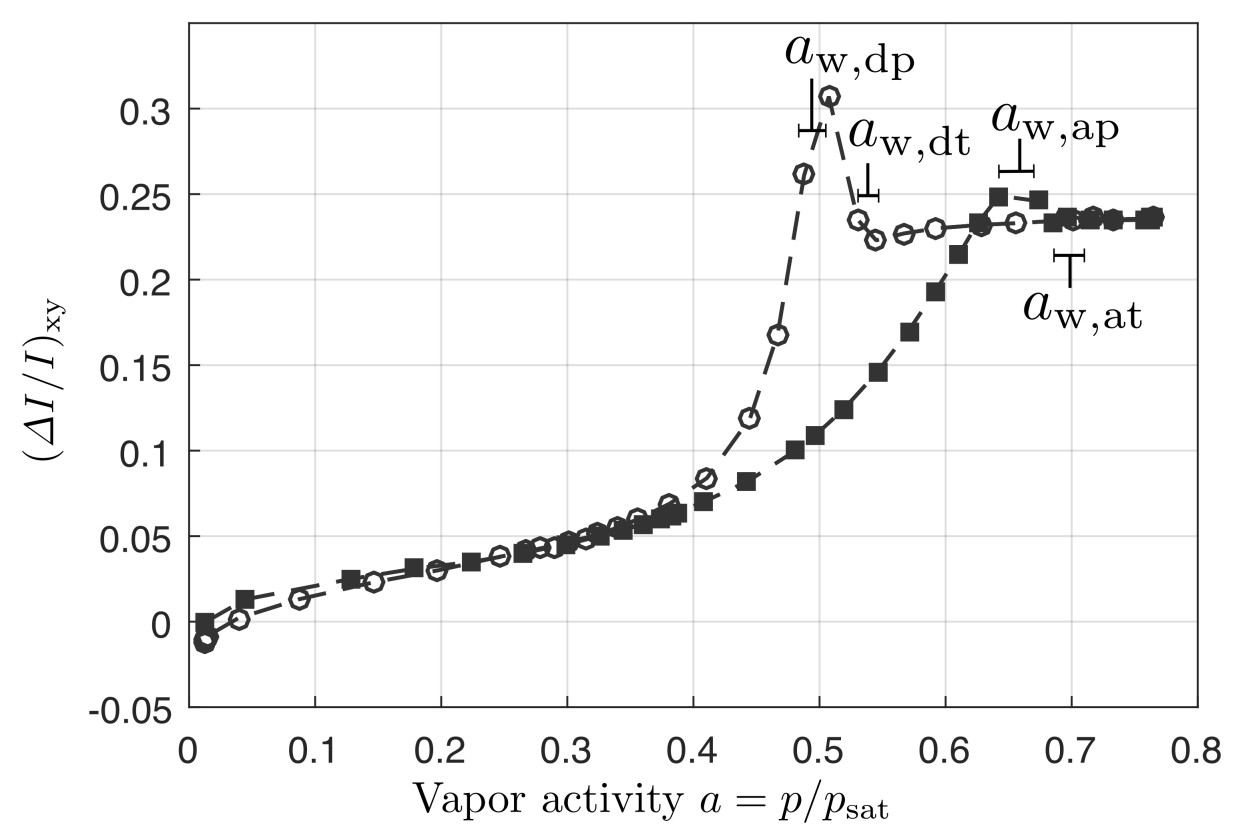

Figure 2: Reflectance isotherms for pure water. Adsorption-tension activity, desorptiontension activity, adsorption-peak activity and desorption-peak activity, as defined in Figure 1 , are referred to as $a_{\mathrm{w}, \text { at }}$ and $a_{\mathrm{w}, \mathrm{dt}}, a_{\mathrm{w}, \text { ap }}$ and $a_{\mathrm{w}, \mathrm{dp}}$, respectively for the special case of pure water. Closed squares and open circles represent adsorption branch and desorption branch, respectively.

Before recording reflectance isotherms for pure water, $\mathrm{NaCl}$ solutions or $\mathrm{LiCl}$ solutions, we prepared the porous silicon sample as follows. The porous silicon substrate was sonicated in mixture of $50 \%(\mathrm{v} / \mathrm{v})$ acetone- $50 \%(\mathrm{v} / \mathrm{v})$ ethanol for 5 minutes and the substrate was dried by blowing it with compressed nitrogen. For isotherms with pure water, the sample was then let to dry in vacuum for 30 minutes. For isotherms with solutions, a solution (1 ml) of known concentration (see Figure 3,4) of LiCl (Alfa Aesar, 99\%) or NaCl (Amresco, 
99.9\% Biotechnology Grade) was poured on porous silicon. Wettability effects (hydrophilic porous silicon vs. hydrophobic surrounding bulk silicon) confined the solution into puddle on the porosified region. The sample was let to imbibe the solution for 30 minutes (diffusion constant of $\mathrm{NaCl}$ in aqueous phase ${ }^{22}$ is of the order of $D \sim 10^{-5} \mathrm{~m}^{2} / \mathrm{s}$, such that time taken for diffusive equilibrium $\left(\equiv L^{2} / D\right)$ of solute in the pore liquid through the thickness, $L \sim 5$ $\mu \mathrm{m}$, of the pores, is on the order of $\mu \mathrm{s}$ ). Excess solution on top of porous silicon was removed using clean room wipe (Bluesorb 750, Berkshire Co.). The sample was let to dry in vacuum for 30 minutes.

We obtained reflectance isotherms (Figures 1(b), 2-4) by placing the prepared sample (previous paragraph) in a vacuum chamber equipped with an optical window; the sample rested on the bottom surface of the chamber which was thermostated at $T=15^{\circ} \mathrm{C}$. Pure water vapor was obtained by evaporation from a degassed liquid water source and flowed through the chamber toward the vacuum pump. We measured pressure of the flowing vapor, $p[\mathrm{~Pa}]$, using a pressure gauge (Pfeiffer Vacuum, CCR362) connected to the chamber. We set the pressure between $p=0$ and $p=p_{\text {sat }}$ by adjusting the relative opening of two needle valves, one upstream and one downstream of chamber. We estimated an uncertainty on the vapor activity, $a=p / p_{\text {sat }}$ of \pm 0.005 from the error of the pressure gauge reading as well as the measured fluctuations of $T$ and $p$ during the experiments.

We started isotherms from low vapor pressure and followed the adsorption branch first. When bulk solution started to appear on the substrate surface (sharp increase in reflected light from the solution droplets on top of the surface), we began to lower the pressure to follow the desorption branch. At each pressure step; we waited 150 seconds (the effective diffusion constant of water vapor in the nanopores as estimated in Vincent et al. ${ }^{20}$ is of the order of $D \sim 10^{-10} \mathrm{~m}^{2} / \mathrm{s}$, such that the time taken for diffusive equilibrium $\left(\equiv L^{2} / D\right)$ of vapor through the thickness, $L \sim 5 \mu \mathrm{m}$, of the pores, is only a fraction of a second) before capturing images for next 30 seconds at a capture rate of 1 frame per second. Images were analyzed as described previously. ${ }^{20}$ Briefly, we recorded time-lapse image sequences of the 
sample using Point Grey Research Grasshopper3 Mono camera fitted with AF Micro-Nikkor $60 \mathrm{~mm} \mathrm{f} / 2.8 \mathrm{D}$ lens. We generated white-light, diffuse illumination with a Schott ACE light source (150 W halogen lamp) operating at $~ 50 \%$ of its maximum intensity. We analyzed the response to changes in vapor pressure by extracting the local relative change in reflectance, $(\Delta I / I)_{i}$, where $(\Delta I / I)_{i}$ was calculated by measuring the change in gray-scale value of pixels as described previously. ${ }^{20}$ Water uptake produced a darkening of the image (images in Fig 1(a)), resulting in $(\Delta I / I)_{i}>0$. In other words, a high $\Delta I / I$ means a dark layer (low reflectance). For the measurement of the reflectance isotherm, we evaluated the average change in intensity $\langle\Delta I / I\rangle=\frac{1}{N} \sum_{i}(\Delta I / I)$ over the whole region of interest $\left(\sim 10 \mathrm{~mm}^{2}\right)$.

We report the mean value and error of vapor activities of interest $\left(a_{\mathrm{s}}, a_{\mathrm{at}}, a_{\mathrm{dt}}, a_{\mathrm{ap}}, a_{\mathrm{dp}}\right.$ and $a_{\mathrm{c}}$ ) by choosing two data points that mark the transition as defined by each of these activities in the introduction and as shown in Figure 1(b).

\section{Theory}

In the following, we present a theoretical approach to interpret how changes in vapor activity correlate to changes in the state of solutions within the porous network. We recall that our direct observations are of optical reflectance; we discuss our interpretation of this optical information in terms of the state of the solution in the pores. In order to interpret the optical isotherms of the solution-filled sample (Figure 1, 3 and 4), we make comparisons to the isotherms observed with pure liquid water in the pores (Figure 2) with the same sample. In this section, we first discuss qualitatively the different regimes of the pore solution as a function of vapor activity, then we develop expressions for the liquid-vapor equilibrium and derive relationships between the case of a solution filling the pores and the pure solvent case. Finally, after considering crystallization with a model of confined crystal nucleation in the pore solution, we discuss the origin of the peaks in scattering observed on the optical isotherms $\left(a_{\mathrm{dp}}\right.$ and $a_{\mathrm{ap}}$ in Figure $\left.1 \mathrm{~b}\right)$. 


\section{Qualitative considerations, assumptions and definitions}

Here, we elaborate qualitatively on the behavior of pores filled with solution as a function of vapor pressure, before quantifying this behavior with a thermodynamic model in the next subsections.

In the following, we define the mole fraction as $x=n_{\mathrm{s}} /\left(n_{\mathrm{s}}+n_{\mathrm{w}}\right)$, where $n_{\mathrm{s}}$ is the amount of substance of undissociated salt and $n_{\mathrm{w}}$ is the amount of substance of water. A solution with mole fraction $x$ has an equilibrium vapor pressure, $p_{\text {sol }}(x)<p_{\text {sat }}$, which decreases below 1 as $x$ increases above 0 , and is a direct consequence of solute content. We define the activity of solution as $a_{\text {sol }}(x)=p_{\text {sol }}(x) / p_{\text {sat }}<1$. By definition, $a_{\text {sol }}(x)$ corresponds to the activity of the vapor with which a bulk sample of the solution with the same mole fraction of solute, $x$, as the solution present in the pores would be in equilibrium, and is thus independent of capillary pressure or confinement. The properties of solution initially used to fill the pores are denoted by the values of the molar fraction as $x_{\mathrm{s}}$ (i.e., $x=x_{\mathrm{s}}$ ) and of the corresponding activity of solution as bulk-solution activity, $a_{\mathrm{s}}\left(a_{\mathrm{sol}}\left(x_{\mathrm{s}}\right)=a_{\mathrm{s}}\right)$.

The classical picture of equilibrium between pure pore liquid and vapor ${ }^{23}$ involves a variation of the shape of the liquid-vapor interface as a function of vapor activity, $a=p / p_{\text {sat }}$. When $a=1$ (vapor saturation), the meniscus is flat as in Figure $1(\mathrm{c})$, while for $a<1$, the meniscus is curved as in Figure 1(d). The curvature increases when $a$ decreases, implying a varying contact angle of the interface with respect to the pore wall (Figure 1(d)), and a negative pressure (mechanical tension) in the liquid due to capillarity. When a reaches a value at which the contact angle reaches its minimum (receding) value, equilibrium becomes impossible and the pore liquid evaporates (desorption).

If the pore is filled with solution, two important qualitative differences appear. First, vapor saturation with respect to the solution does not occur at $a=1$, but at a value, $a_{\mathrm{s}}<1$, that decreases with increasing solute concentration; when $a=a_{\mathrm{sol}}=a_{\mathrm{s}}$, the situation is as depicted in Figure 1(c), while $a<a_{\mathrm{s}}$ corresponds to the varying contact angle regime of 
Figure $1(\mathrm{~d})$. Second, when the meniscus contact angle $\theta_{\text {sol }}$ reaches its receding value $\left(\theta_{\text {rec }}\right.$, Figure 1(e)), a further decrease of the vapor pressure does not induce complete evaporation of the liquid in the pore, because receding of the meniscus results in an increase of the solute concentration, allowing the establishment of a new equilibrium between the solution and the vapor by lowering the chemical potential of the solvent (Figure 1(f)). Decreasing the vapor pressure thus results in a continuous decrease of the volume of solution in the sample, until concentration is large enough to induce crystallization of the solute (Figure 1(j)).

In other words, while a pure liquid in a pore only has one degree of freedom (the contact angle with the pore wall) to achieve equilibrium with vapor, a solution has both contact angle $\left(\theta_{\text {sol }}\right)$ and concentration (mole fraction, $\left.x\right)$ as degrees of freedom, resulting in a larger range of possible equilibria, and a more continuous evaporation process from the medium. At high vapor pressure (Figure 1(d)), the solution fills the pore completely, and only the contact angle varies with $a$; changes in volume induced by this change in contact angle are negligible for large enough systems, so that the concentration of solute is essentially constant in that regime (varying $\theta_{\text {sol }}$, constant $x=x_{\mathrm{s}}$ ). At lower vapor pressures (Figure $1(\mathrm{f})$ ), another regime is reached where the volume of solution in the pore adapts to the imposed vapor pressure while the contact angle is equal to its receding value that we assume independent of solute concentration (constant $\theta_{\text {sol, }}$ varying $x$ ). The transition between these regimes occurs at $a=a_{\mathrm{dt}}$ (Figure 1(e)), the desorption-tension activity at which the meniscus curvature (and thus the mechanical tension in the liquid) becomes maximum when decreasing the vapor pressure.

A similar transition occurs on the adsorption branch, i.e. upon increasing the vapor pressure: following deliquescence of the crystal, equilibration of the confined solution with increasing vapor pressure proceeds through dilution of the solution (Figure $1(\mathrm{~h})$ ), which expands with its advancing contact angle, $\theta_{\text {adv }}$, until filling the pore completely at the adsorption-tension activity, $a_{\text {at }}$ (Figure 1(i)). Above $a_{\text {at }}$, a regime of constant $x$ and varying $\theta_{\text {sol }}$ is recovered (Figure $\left.1(\mathrm{j})\right)$. Here, we use an elementary picture of a contact angle differ- 

and account in a simple manner for desorption-adsorption hysteresis; we do not attempt to model other phenomena such as pore-blocking ${ }^{23,24}$ or to investigate the physical origins of the hysteresis in our porous network. We also assume that the advancing/receding contact angles, $\theta_{\mathrm{adv}(\mathrm{rec})}$, do not depend on the composition of the solution, and are the same for pure water $(x=0)$ and for solution (any $x)$, i.e., $\left.\theta_{\text {adv }}\right|_{\text {water }}=\left.\theta_{\text {adv }}\right|_{\text {solution }}$ and $\left.\theta_{\text {rec }}\right|_{\text {water }}=\left.\theta_{\text {rec }}\right|_{\text {solution }}$.

In the following, we assume that the liquid within the pores behaves like a bulk substance with respect to solution thermodynamics and liquid-vapor equilibrium. Our previous work with liquid water in the same porous medium tends to support this assumption. ${ }^{20,21}$ More generally, one only expects strong non-continuum effects for pore diameters below $1 \mathrm{~nm} ;{ }^{25}$ pore diameters in the present study are $\sim 3 \mathrm{~nm}$. With the experiments presented here with aqueous solutions, we confront this hypothesis and find it satisfactory. While nanoscale confinement might make the pore liquid deviate from its bulk behavior, we do not resolve such effects with the measurements presented here.

\section{Kelvin-Laplace relation for pure solvent}

We begin with a reminder of the simplest case of a pure solvent (water) in a porous medium in equilibrium with its vapor. We have previously reported on optical isotherms for pure silicon with pure water (Figure 2); ${ }^{20}$ here, this case serves as a reference to aid in the interpretation of isotherms for aqueous solutions. Throughout, we adopt an idealized perspective on the character of the pores: we treat them as cylindrical pores of uniform radius, $r_{\mathrm{p}}$, for the ease of subsequent derivation of capillary pressure. Equilibrium is established between bulk liquid and pure vapor at saturation pressure, $p_{\text {sat }}(T)$, for a given temperature, $T$. For pure water, we use the equation of state of Wagner et al. ${ }^{26}$ for the saturation pressure, $p_{\text {sat }}(T)$. However, when the liquid in the pores is exposed to sub-saturated vapor, thermodynamic equilibrium is established when chemical potential of water, $\mu_{\mathrm{w}}^{\mathrm{liq}}[\mathrm{J} / \mathrm{mol}]$, in pores at pressure, $P$, is equal to the chemical potential of the imposed vapor, $\mu_{\mathrm{w}}^{\mathrm{vap}}$ at pressure, $p$, for a given temperature, 
$T$

$$
\mu_{\mathrm{w}}^{\operatorname{liq}}(P, T)=\mu_{\mathrm{w}}^{\mathrm{vap}}(p, T)
$$

With chemical potential of bulk liquid in equilibrium with vapor $\left(P=p=p_{\text {sat }}\right), \mu_{\mathrm{o}}(T)$, as reference, chemical potential of pore liquid and vapor is found upon integrating the isothermal Gibbs-Duhem equation:

$$
\begin{gathered}
\mu_{\mathrm{w}}^{\mathrm{liq}}(P, T)=\mu_{\mathrm{o}}(T)+v_{\mathrm{w}}^{\mathrm{liq}}\left(P-p_{\mathrm{sat}}\right) \\
\text { and } \\
\mu_{\mathrm{w}}^{\mathrm{vap}}(p, T)=\mu_{\mathrm{o}}(T)+R T \ln \left(\frac{p}{p_{\mathrm{sat}}}\right),
\end{gathered}
$$

where $R=8.314[\mathrm{~J} / \mathrm{K}]$ is the universal gas constant and $v_{\mathrm{w}}^{\text {liq }}\left[\mathrm{m}^{3} / \mathrm{mol}\right]$ is the molar volume of liquid water at temperature $T$. In Equation 2, we assume $v_{\mathrm{w}}^{\text {liq }}$ to be independent of pressure, i.e., that the liquid is incompressible; in Equation 3, we assume that the vapor is an ideal gas. Equation 1 combined with 2 and 3 yield the Kelvin equation:

$$
P=p_{\mathrm{sat}}+\frac{R T}{v_{\mathrm{w}}^{\operatorname{liq}}} \ln \left(\frac{p}{p_{\mathrm{sat}}}\right) .
$$

The pressure difference between pore liquid and vapor is mechanically balanced by the capillary pressure at the liquid vapor meniscus as given by the Young-Laplace:

$$
P-p=-\frac{2 \gamma_{\mathrm{w}} \cos \left(\theta_{\mathrm{w}}\right)}{r_{\mathrm{p}}}
$$

where $\gamma_{\mathrm{w}}[\mathrm{N} / \mathrm{m}]$ is surface tension at the solution-vapor interface, $\theta_{\mathrm{w}}$ is the angle the solvent makes with the pore wall and $r_{\mathrm{p}}$ is the pore radius. In practice, the pressure, $P$, in pore liquid, predicted by Kelvin equation (Equation 4) is much larger in magnitude $(P \sim \mathrm{MPa})$ than the vapor pressure or saturation vapor pressure $\left(p, p_{\text {sat }} \sim \mathrm{kPa}\right)$ such that Equations 4 
and 5 can be rewritten, to an excellent approximation, as:

$$
-\frac{2 \gamma_{\mathrm{w}} \cos \left(\theta_{\mathrm{w}}\right)}{r_{\mathrm{p}}}=\frac{R T}{v_{\mathrm{w}}^{\operatorname{liq}}} \ln \left(\frac{p}{p_{\mathrm{sat}}}\right)=\frac{R T}{v_{\mathrm{w}}^{\operatorname{liq}}} \ln \left(a_{\mathrm{w}}\right) .
$$

In Equation 6, we denote the vapor activity imposed on the sample filled with pure water as: $a_{\mathrm{w}}=\frac{p}{p_{\mathrm{sat}}}$. This notation allows us to distinguish the pure solvent case from the solution case as we develop relationships between the two in the following sub-sections.

For the special case considered here, where pore liquid is pure water, i.e., $a_{\mathrm{s}}=1$, we rewrite $a_{\text {at }}$ and $a_{\mathrm{dt}}$ as $a_{\mathrm{w}, \mathrm{at}}(=0.698 \pm 0.019)$ and $a_{\mathrm{w}, \mathrm{dt}}(=0.533 \pm 0.018)$ respectively (Figure 2 ), for clarity in subsequent derivations. In Equation 6, contact angle, $\theta_{\mathrm{w}}$ is a function of vapor activity, $a_{\mathrm{w}}$, for vapor activity $a_{\mathrm{w}} \geq a_{\mathrm{w}, \text { at }}$ on the adsorption branch and $a_{\mathrm{w}} \geq a_{\mathrm{w}, \mathrm{dt}}$ on the desorption branch (analogous to Figure 1(e, i) for pure solvent). At $a=a_{\mathrm{w}, \text { at }}$ or $a=a_{\mathrm{w}, \mathrm{dt}}$, the solvent fills the pores and the contact angle equals the equilibrium advancing angle $\theta_{\mathrm{w}}=\theta_{\mathrm{adv}}$ or the equilibrium receding angle, $\theta_{\mathrm{w}}=\theta_{\mathrm{rec}}$ respectively and hence, at vapor activity, $a=a_{\mathrm{w}, \mathrm{at}(\mathrm{dt})}$, Equation 6 can be rewritten as:

$$
-\frac{2 \gamma_{\mathrm{w}} \cos \left(\theta_{\mathrm{adv}(\mathrm{rec})}\right)}{r_{\mathrm{p}}}=\frac{R T}{v_{\mathrm{w}}} \operatorname{liq}\left(a_{\mathrm{w}, \mathrm{at}(\mathrm{dt})}\right) .
$$

We elaborated on the distinction between the advancing contact angle and receding contact angle earlier in this section.

\section{Kelvin-Laplace relation for solutions}

Now, we adapt the same set of equations to the multicomponent case of solutions. We consider a situation as shown in Figure 1(d-f, h-j), where two-phase equilibrium exists between pore-solution and water vapor at temperature, $T$, with the solution at pressure, $P$, and water vapor at pressure, $p$, respectively. Equilibrium is established when,

$$
\mu_{\mathrm{w}}^{\mathrm{sol}}(P, T, x)=\mu_{\mathrm{w}}^{\mathrm{vap}}(p, T)
$$


where $\mu_{\mathrm{w}}^{\mathrm{sol}}(P, T, x)$ is the chemical potential of water in solution at pressure $P$, temperature, $T$, and mole fraction, $x$, and $\mu_{\mathrm{w}}^{\mathrm{vap}}(p, T)$ is the chemical potential of water vapor at pressure, $p$, and temperature, $T$. Integrating the isothermal Gibbs-Duhem equation with saturation pressure of pure water, $p_{\text {sat }}(T)$, as the reference pressure, and with the chemical potential of bulk, pure water and vapor $\left(\mu_{\mathrm{o}}\right)$ as reference, the chemical potential of liquid water in solution and vapor can be written as: ${ }^{27}$

$$
\begin{gathered}
\mu_{\mathrm{w}}^{\mathrm{sol}}(P, T, x)=\mu_{\mathrm{o}}(T)+R T \ln \left(\frac{p_{\mathrm{sol}}(x)}{p_{\mathrm{sat}}}\right)+v_{\mathrm{w}}^{\mathrm{sol}}\left(P-p_{\mathrm{sol}}\right) \\
=\mu_{\mathrm{o}}(T)+v_{\mathrm{w}}^{\mathrm{sol}}\left(P-\Pi(x)-p_{\mathrm{sat}}\right) \\
\text { and } \\
\mu_{\mathrm{w}}^{\mathrm{vap}}(p, T)=\mu_{\mathrm{o}}+R T \ln \left(\frac{p}{p_{\mathrm{sat}}}\right)
\end{gathered}
$$

where $\Pi(x)=\frac{-R T}{v_{\mathrm{w}}^{\text {sol }}} \ln \left(\frac{p_{\mathrm{sol}}(x)}{p_{\mathrm{sat}}}\right)+p_{\mathrm{sol}}-p_{\text {sat }}$ is the osmotic pressure of the solution (assuming $\left.v_{\mathrm{w}}^{\mathrm{sol}} \approx v_{\mathrm{w}}^{\text {liq }}\right),{ }^{27} p_{\mathrm{sol}}(x)$ is the vapor pressure of solution with dissolved mole fraction of solute $x$, and $v_{\mathrm{w}}^{\mathrm{sol}}\left[\mathrm{m}^{3} / \mathrm{mol}\right]$ is the molar volume of the water component of the pore solution at temperature $T$ (assuming $v_{\mathrm{w}}^{\text {sol }}$ to be independent of pressure, i.e., considering the liquid as incompressible). On defining activity of water vapor, $a=p / p_{\text {sat }}$ (also referred to as relative humidity), and the activity of solution with dissolved mole fraction of solute $x$, as, $a_{\mathrm{sol}}(x)=p_{\mathrm{sol}}(x) / p_{\mathrm{sat}}$, at equilibrium $\left(\mu_{\mathrm{w}}^{\mathrm{sol}}(P, T, x)=\mu_{\mathrm{w}}^{\mathrm{vap}}(p, T)\right)$, Equations 8,9 and 10 yield

$$
\begin{aligned}
P & =p_{\text {sat }}+\Pi(x)+\frac{R T}{v_{\mathrm{w}}^{\mathrm{sol}}} \ln \left(\frac{p}{p_{\mathrm{sat}}}\right) \\
& =p_{\mathrm{sol}}+\frac{R T}{v_{\mathrm{w}}^{\mathrm{sol}}} \ln \left(\frac{p}{p_{\mathrm{sol}}(x)}\right) \\
& =p_{\mathrm{sol}}+\frac{R T}{v_{\mathrm{w}}^{\mathrm{sol}}} \ln \left(\frac{a}{a_{\mathrm{sol}}(x)}\right) .
\end{aligned}
$$


The pressure difference between the solution and the water vapor is again mechanically balanced by the capillary pressure at the liquid-vapor meniscus as given by Young-Laplace:

$$
P-p=-\frac{2 \gamma_{\mathrm{sol}}(x) \cos \left(\theta_{\mathrm{sol}}\right)}{r_{\mathrm{p}}}
$$

where $\gamma_{\text {sol }}[\mathrm{N} / \mathrm{m}]$ is surface tension at the solution-vapor interface, $\theta_{\text {sol }}$, as seen in Figure $1(\mathrm{~d}$, $\mathrm{j}$ ), is the equilibrium angle the solution makes with the pore wall and $r_{p}$ is the pore radius. Neglecting again the solution pressure, $P$, which is much larger in magnitude $(P \sim \mathrm{MPa})$ than the vapor pressure or saturation vapor pressure $\left(p, p_{\text {sat }} \sim \mathrm{kPa}\right)$, Equations 11 and 12 can be combined to a good approximation, as:

$$
-\frac{2 \gamma_{\mathrm{sol}}(x) \cos \left(\theta_{\mathrm{sol}}\right)}{r_{\mathrm{p}}}=\frac{R T}{v_{\mathrm{w}}^{\mathrm{sol}}} \ln \left(\frac{a}{a_{\mathrm{sol}}(x)}\right) .
$$

We account for the concentration dependence of surface tension, $\gamma_{\text {sol }}$ (see SI, Figure S1), and the activity of solution, $a_{\text {sol }}$ (see Supplementary Information(SI), Figure S2 and S3).

We now rewrite Equation 13 to denote the two regimes elaborated earlier on the variation in concentration of solution with vapor activity: at high vapor pressure (Figure 1 (c, d, e, $\mathrm{i}, \mathrm{j})$ ), the porous medium remains full of solution and only the contact angle varies with $a$ (constant $x$, varying $\theta_{\text {sol }}$ and varying $p$ ); at low vapor pressure (Figure $1(\mathrm{f}, \mathrm{h})$ ), the solution recedes or advances where the concentration of solution varies with $a$ (constant $\theta_{\text {sol }}$, varying $x$ and varying $p)$.

For higher vapor activity (Figure 1 (c, d, e, i, j)), the concentration of pore solution remains constant while the contact angle changes with changing vapor activity. The bulk solution (state when pores are entirely filled, Figure 1(c, d, e, i, j)) is characterized by bulksolution activity, $a_{\mathrm{s}}$, i.e, $a_{\mathrm{s}}=a_{\mathrm{sol}}\left(x_{\mathrm{s}}\right)=p_{\mathrm{s}} / p_{\text {sat }}(T)$, with the vapor pressure of the bulk solution, $p_{\mathrm{sol}}\left(x_{\mathrm{s}}\right)=p_{\mathrm{s}}$ and dissolved mole fraction of solute, $x=x_{\mathrm{s}}$. Note: we calculate $x_{\mathrm{s}}$ from the experimental values of bulk-solution activity, $a_{\mathrm{s}}$ using empirical relations from the literature between $a_{\text {sol }}$ and $x$ (see Supplementary Information (SI) for empirical rela- 
tions). ${ }^{28,29}$ As described earlier, the activity of the solution $\left(=a_{\mathrm{s}}\right)$ and the concentration of solution remains constant $\left(=x_{\mathrm{s}}\right)$ for the range of vapor activity as shown in Figure 1 (c, d, e, $\mathrm{i}, \mathrm{j}$ ) while the contact angle, $\theta_{\text {sol }}$ varies as a function of $a$, and, hence, Equation 13 can be rewritten as:

$$
-\frac{2 \gamma_{\mathrm{sol}}\left(x_{\mathrm{s}}\right) \cos \left(\theta_{\mathrm{sol}}\right)}{r_{\mathrm{p}}}=\frac{R T}{v_{\mathrm{w}}^{\mathrm{sol}}} \ln \left(\frac{a}{a_{\mathrm{s}}}\right)
$$

and, at special case of vapor activity $a=a_{\mathrm{at}}$ and vapor activity $a=a_{\mathrm{dt}}$, the contact angle equals the equilibrium advancing angle, i.e, $\theta_{\text {sol }}=\theta_{\text {adv }}$ (Figure $1(\mathrm{f})$ ) on the adsorption branch and equilibrium receding angle, i.e, $\theta_{\text {sol }}=\theta_{\text {rec }}($ Figure $1(\mathrm{~h}))$ on the desorption branch, and hence, Equation 14 can be rewritten as:

$$
-\frac{2 \gamma_{\mathrm{sol}}\left(x_{\mathrm{s}}\right) \cos \left(\theta_{\mathrm{adv}(\mathrm{rec})}\right)}{r_{\mathrm{p}}}=\frac{R T}{v_{\mathrm{w}}^{\mathrm{sol}}} \ln \left(\frac{a_{\mathrm{at}(\mathrm{dt})}}{a_{\mathrm{s}}}\right) .
$$

We use Equation 15 in the next subsection to predict evolution of $a_{\text {at(dt) }}$ with $a_{\mathrm{s}}$.

For lower vapor activity $\left(a<a_{\text {at }}\right.$ on the adsorption branch and $a<a_{\mathrm{dt}}$ on the desorption branch) as shown Figure 1(f, h), the concentration of pore solution changes $\left(x>x_{\mathrm{s}}\right)$ while the contact angle remains constant at equilibrium advancing angle, i.e, $\theta_{\text {sol }}=\theta_{\text {adv }}$ (Figure $1(\mathrm{~h})$ ) on the adsorption branch and equilibrium receding angle, i.e, $\theta_{\text {sol }}=\theta_{\text {rec }}($ Figure $1(\mathrm{f}))$ on the desorption branch and and hence, Equation 13 can be rewritten as:

$$
-\frac{2 \gamma_{\mathrm{sol}}(x) \cos \left(\theta_{\mathrm{adv}(\mathrm{rec})}\right)}{r_{\mathrm{p}}}=\frac{R T}{v_{\mathrm{w}}^{\mathrm{sol}}} \ln \left(\frac{a}{a_{\mathrm{sol}}(x)}\right) .
$$

From Equation 16, we infer that for lower vapor activities $\left(a<a_{\text {at }}\right.$ on the adsorption branch and $a<a_{\mathrm{dt}}$ on the desorption branch), the concentration of the pore solution, $x$, is only a function of imposed vapor activity and independent of the bulk-solution activity, $a_{\mathrm{s}}$, i.e, the activity of solution when the pores are entirely filled. 


\section{Relationship between pure solvent and solution isotherms}

Here, we derive a relation between the pure solvent and solution isotherms by combining the Kelvin-Laplace equations for pure solvent (Equation 6) with that for the solution (Equation 15) to predict the vapor activity associated with the onset of desorption $\left(a_{\mathrm{dt}}\right)$ and end of adsorption $\left(a_{\mathrm{at}}\right)$ (Figure 5). This derivation assumes an idealized case of single pore with no non-continuum effects. By combining Equations 7 and 15, we derive the following relation that is independent of $r_{\mathrm{p}}$ :

$$
a_{\mathrm{at}(\mathrm{dt})}=a_{\mathrm{s}} \times a_{\mathrm{w}, \mathrm{at}(\mathrm{dt})}^{\gamma_{\mathrm{sol}}\left(x_{\mathrm{s}}\right) v_{\mathrm{w}}^{\mathrm{sol}} / \gamma_{\mathrm{w}} v_{\mathrm{w}}^{\text {liq }}}
$$

Equation 17 serves to predict the evolution of important features noted in Figure 1(b) as a function of $a_{\mathrm{s}}$.

We assume that the molar volume of water is independent of concentration of solute, i.e., $v_{\mathrm{w}}^{\mathrm{sol}} \approx v_{\mathrm{w}}^{\text {liq }},{ }^{27}$ Equation 17 yields:

$$
a_{\mathrm{at}(\mathrm{dt})}=a_{\mathrm{s}} \times a_{\mathrm{w}, \mathrm{at}(\mathrm{dt})}^{\gamma_{\mathrm{sol}}\left(x_{\mathrm{s}}\right) / \gamma_{\mathrm{w}}}
$$

We account for the concentration-dependence of $\gamma_{\text {sol }}$ as follows: we calculate the concentration, $x_{\mathrm{s}}$, from the experimental values of $a_{\mathrm{s}}\left(=a_{\text {sol }}\left(x_{\mathrm{s}}\right)\right)$, using empirical relations between $a_{\text {sol }}$ and $x$ (see Supplementary Information (SI), Figure S2 and S3) and then calculate $\gamma_{\text {sol }}$ at concentration, $x_{\mathrm{s}}$, from the known empirical relation between $\gamma_{\text {sol }}$ and $x$ (see SI, Figure S1). Figure 5 (dashed lines) presents theoretical predictions of $a_{\mathrm{at}}$ and $a_{\mathrm{dt}}$ from Equation 18. We highlight the effect of concentration-dependent surface tension, $\gamma_{\text {sol }}\left(x_{\mathrm{s}}\right)$, by comparing it with the predicted $a_{\text {at }}$ and $a_{\mathrm{dt}}$ upon assuming a constant surface tension $\left(\gamma_{\mathrm{sol}} \approx \gamma_{\mathrm{w}}\right)$ in SI (Figure S8). 


\section{Classical Nucleation Theory (CNT) for crystallization in nanopores}

In the following, we adapt the theory proposed by Fukuta ${ }^{30}$ for freezing of a pore liquid to the context of solute crystallization. This theory accounts for the reduced pressure in the pore liquid due to metastable equilibrium with the unsaturated vapor. We consider here homogeneous salt crystallization in a supersaturated solution in a cylindrical pore, as sketched in Figure 1(g). We construct this theory by hypothesizing a homogeneous mode of nucleation in order to estimate the theoretical supersaturation needed for homogeneous crystallization in a nanopore. We do not rule out the possibility for other modes of nucleation. According to CNT, for homogeneous nucleation of a salt crystal in a supersaturated solution, there is a critical population of salt molecules that act as embryo for crystallization. We assume that the kinetics of crystallization is fast and that crystallization is triggered as soon as the formation and growth of this critical nucleus is sterically allowable. We discuss the validity of this assumption as we discuss results (see Results section). The change in free energy, $\Delta G$, for formation of a spherical crystal of radius, $r$, includes contributions associated with the bulk phases (solution + crystal) and the interface between the two:

$$
\Delta G=\frac{\frac{4}{3} \pi r^{3}}{v_{\mathrm{s}}^{\mathrm{c}}}\left(\mu_{\mathrm{s}, \mathrm{r}_{\mathrm{p}}}^{\mathrm{c}}-\mu_{\mathrm{s}, \mathrm{r}_{\mathrm{p}}}^{\mathrm{sol}}\right)+4 \pi r^{2} \gamma_{\ell \mathrm{c}}
$$

where $v_{\mathrm{s}}^{\mathrm{c}}\left[\mathrm{m}^{3} /\right.$ mole $]$ is the molar volume of salt crystal, $\gamma_{\ell c}\left[\mathrm{~J} / \mathrm{m}^{2}\right]$ is the surface tension at the interface of two phases (for $\mathrm{NaCl}$, we use $\gamma_{\ell c}=0.1 \mathrm{~J} / \mathrm{m}^{2}$; for $\mathrm{LiCl}$, we use $\gamma_{\ell c}=$ $\left.0.12 \mathrm{~J} / \mathrm{m}^{2}\right) ;{ }^{31,32} \mu_{\mathrm{s}, \mathrm{r}_{\mathrm{p}}}^{\mathrm{c}}$ is the chemical potential of a spherical solute crystal in a pore of radius, $r_{\mathrm{p}}$, and $\mu_{\mathrm{s}, \mathrm{r}_{\mathrm{p}}}^{\mathrm{sol}}$ is the chemical potential of dissolved solute in a pore of radius, $r_{\mathrm{p}} \cdot{ }^{33-35}$ Finding the maximum of $\Delta G$ with Equation 19 gives the critical radius, $r_{\mathrm{c}}$, in terms of chemical potential as,

$$
r_{\mathrm{c}}=\frac{-2 \gamma_{\ell \mathrm{c}} v_{\mathrm{s}}^{\mathrm{c}}}{\mu_{\mathrm{s}, \mathrm{r}_{\mathrm{p}}}^{\mathrm{c}}-\mu_{\mathrm{s}, \mathrm{r}_{\mathrm{p}}}^{\mathrm{sol}}} .
$$

The activity of unsaturated vapor regulates the dissolved mole fraction, $x$, and hence, the supersaturation of solution (in other words, activity of solute and solvent) for two-phase 
equilibrium (see Equation 16). According to classical nucleation theory, a nucleus grows when the size of nucleus is greater than the critical radius, $r_{\mathrm{c}}$, and the pore radius determines the maximum size of spherical nucleus. Hence, by employing the constraint on the size of critical nucleus due to confinement, $r_{\mathrm{c}} \leq r_{\mathrm{p}}$, we can find a relationship for the theoretical mole fraction of dissolved solute needed for the onset of crystallization, $x_{\mathrm{c}, \mathrm{th}}$, equivalently, the theoretical supersaturation of dissolved solute, $x_{\mathrm{c}, \mathrm{th}} / x_{\mathrm{sat}}$, for onset of crystallization from chemical and mechanical equilibrium relations substituted in Equation 20 in terms of measurable and known parameters (see SI):

$$
r_{\mathrm{c}}=r_{\mathrm{p}}=\frac{2 \gamma_{\ell \mathrm{c}} v_{\mathrm{s}}^{\mathrm{c}}}{\left(v_{\mathrm{s}}^{\mathrm{c}}-v_{\mathrm{s}}^{\mathrm{sol}}\right) \frac{2 \gamma_{\mathrm{sol}} \cos \left(\theta_{\mathrm{rec}}\right)}{r_{\mathrm{p}}}+\nu R T \ln \frac{\zeta x_{\mathrm{c}, \mathrm{th}}}{\zeta_{\mathrm{sat}} x_{\mathrm{sat}}}} .
$$

where $x_{\text {sat }}$ is the mole fraction of dissolved solute in saturated bulk solution, $\zeta_{\text {sat }}$ is the activity coefficient of solute in saturated bulk solution, $\zeta$ is the activity coefficient of solute (see definition in SI), $\nu$ is the number of ions released upon dissociation (for both $\mathrm{NaCl}$ and $\mathrm{LiCl}$, we take $\nu=2), v_{\mathrm{s}}^{\text {sol }}$ is the partial molar volume of solute in solution and $\gamma_{\mathrm{sol}}$ is the surface tension of the solution-vapor interface. We can rewrite Equation 21 in terms of theoretical supersaturation needed for the onset of crystallization:

$$
\frac{x_{\mathrm{c}, \mathrm{th}}}{x_{\mathrm{sat}}}=\frac{\zeta_{\mathrm{sat}}}{\zeta} \exp \left[\frac{1}{\nu R T}\left(\frac{2 \gamma_{\ell \mathrm{c}} v_{\mathrm{s}}^{\mathrm{c}}}{r_{\mathrm{p}}}+\frac{2 \gamma_{\mathrm{sol}} \cos \left(\theta_{\mathrm{rec}}\right)}{r_{\mathrm{p}}}\left(v_{\mathrm{s}}^{\mathrm{sol}}-v_{\mathrm{s}}^{\mathrm{c}}\right)\right)\right] .
$$

To evaluate the properties in Equation 22, we adopt the following empirical relations in terms of the mole fraction of solute $(x)$ : solution vapor pressure, $p_{\mathrm{s}}(x),{ }^{28,29}$ surface tension at the solution vapor interface, $\gamma_{\text {sol }}(x),{ }^{28,36}$ the molar volume of the solution, ${ }^{28,37}$ and the activity coefficient of $\mathrm{LiCl}$ and $\mathrm{NaCl}$ solutions, $\zeta(x),{ }^{38,39}$ at a given temperature (see $\mathrm{SI}$ ). Here, we choose $\theta_{\text {rec }} \simeq 0^{\circ}$; we show that the difference in predicted supersaturation across an experimentally reasonable range $\left(0^{\circ}\right.$ to $\left.25^{\circ}\right)$ is $<5 \%$; we plot a comparison in SI, Figure S9. Using this information, we solve Equation 22 numerically for the value of $x_{\mathrm{c}, \mathrm{th}}$.

From Equation 22, we infer that the onset of crystallization occurs at a fixed value of 
dissolved mole fraction of solute, $x_{\mathrm{c}, \mathrm{th}}$, and is independent of the initial mole fraction of solute in the bulk pore solution, $x_{\mathrm{s}}$. Since, $x$ is only a function of vapor activity, $a$, (low vapor activity regime, Equation 16), crystallization should be appearing at the same vapor activity $a$, independent of bulk-solution activity, $a_{\mathrm{s}}$, and $x_{\mathrm{s}}$.

The first term in the exponential on the right hand side of Equation 22 accounts for the unfavorable energetics of forming a critical nucleus of radius, $r_{\mathrm{c}}$, due to the interfacial energy of the crystal in solution $\left(\gamma_{\ell c}\right)$; this term appears in CNT for crystallization from a bulk solution. The second term in the exponential accounts for the impact of the capillary pressure in the pore liquid associated with the liquid-vapor interface, due to equilibrium with an unsaturated vapor as expressed in Equation 13: for an unsaturated vapor, the pressure in solution is lowered; for a positive change in the partial molar volume of the solute upon crystallization $\left(v_{\mathrm{s}}^{\mathrm{c}}>v_{\mathrm{s}}^{\mathrm{sol}}\right)$, this reduction in pressure favors crystallization. ${ }^{30}$ This prediction agrees with the intuitive result from Le Chatelier's principle: growing the crystal with a positive change in molar volume will tend to counteract the tension imposed on the pore liquid by equilibration with an unsaturated vapor. For $\mathrm{LiCl}$, the difference in molar volume of crystal and dissolved solute (see SI, Figure S7) $\left(v_{\mathrm{s}}^{\mathrm{sol}}-v_{\mathrm{s}}^{\mathrm{c}}>0\right)$ opposes crystallization at lower supersaturation and favors crystallization $\left(v_{\mathrm{s}}^{\mathrm{sol}}-v_{\mathrm{s}}^{\mathrm{c}}<0\right)$ at higher supersaturation (see Figure $\mathrm{S} 7)$. However, for $\mathrm{NaCl}$, this quantity is always favorable $\left(v_{\mathrm{s}}^{\mathrm{sol}}-v_{\mathrm{s}}^{\mathrm{c}}<0\right)$ for crystallization (see SI, Figure S7). Note that the magnitude of this effect of negative volume of mixing decreases with increasing supersaturation (see SI Figure S7).

\section{Peaks in scattering}

Here, we develop our hypothesis for the peaks observed on adsorption and desorption branch of the reflectance isotherms (Figures 1 (b), 3, 4). As discussed by Page et al. ${ }^{17}$ and other authors, ${ }^{18}$ draining of a disordered pore network upon desorption can result in the formation of gas and liquid pockets spanning a large number of pores due to pore blocking and associated percolation effects. Close to the percolation threshold, the size, $d$, of these clusters can 
become comparable to the wavelength of light $\lambda_{\text {vis }}$ (Figure $7(\mathrm{a})$ ), resulting in light scattering. A similar but typically weaker effect exists upon adosrption. ${ }^{18}$ As in our previous work, ${ }^{20}$ we attribute the observed peaks in $\langle\Delta I / I\rangle$, corresponding to minima in reflectance, to this percolation and scattering phenomenon; we note that we also observe here a weaker effect on condensation compared to desorption (see Figure 1(b) and 2).

As a percolation-based phenomenon, we expect this effect to be strongly linked to the amount of filling of the network, with maximum scattering at the filling fraction corresponding to the percolation-threshold. Interestingly, there is a way to estimate the filled volume fraction, $\phi$, as a function of imposed vapor activity, $a$, in our experiments.

Indeed, as discussed previously in the Theory section, lowering the vapor activity, $a$, above a pore network filled with a solution results in a progressive emptying of the pore network, as the concentration of the solution increases to maintain equilibrium with the imposed vapor pressure. As a result, any given value of $a$ during desorption corresponds to a well-defined solute fraction $x$, as expressed mathematically in Equation 16. Given the total amount of solute imposed by the initial concentration (equivalently, $a_{\mathrm{s}}$ ), a filled volume fraction can be directly estimated from $a_{\mathrm{s}}$ and $a$. We expect peaks of maximum scattering (lowest reflectance) to occur at similar values of $\phi$ across experiments at different values of $a_{\mathrm{s}}$

Using Kelvin-Laplace relation for pure solvent (Equation 7) and solution (Equation 16) for the case when vapor activity $a<a_{\text {at }}$ on the adsorption branch and $a<a_{\mathrm{dt}}$ on the desorption branch, we derive a relation independent of $r_{\mathrm{p}}$ :

$$
a=a_{\mathrm{sol}}(x) \times a_{\mathrm{w}, \mathrm{at}(\mathrm{dt})}^{\gamma_{\mathrm{sol}}(x) v_{\mathrm{w}}^{\mathrm{sol}} / \gamma_{\mathrm{w}} v_{\mathrm{w}}^{\mathrm{liq}}}
$$

and with the same assumption as made previously, i.e, the molar volume of water is independent of concentration of solute, i.e., $v_{\mathrm{w}}^{\mathrm{sol}} \approx v_{\mathrm{w}}^{\mathrm{liq}},{ }^{27}$ Equation 23 yields:

$$
a=a_{\mathrm{sol}}(x) \times a_{\mathrm{w}, \mathrm{at}(\mathrm{dt})}^{\gamma_{\mathrm{sol}}(x) / \gamma_{\mathrm{w}}}
$$


On the adsorption branch, we denote the vapor activity at the maximum $\langle\Delta I / I\rangle$ (lowest reflectance) by $a_{\text {ap }}\left(<a_{\text {at }}\right)$, referred hereafter as adsorption-peak activity; on the desorption branch, we use $a_{\mathrm{dp}}\left(<a_{\mathrm{dt}}\right)$, referred hereafter as desorption-peak activity (Figure 5). We use Equation 24 to calculate the dissolved mole fraction of solute, $x=x_{\mathrm{ap}}$ and $x=x_{\mathrm{dp}}$ at vapor activity, $a=a_{\mathrm{ap}}$ and $a=a_{\mathrm{dp}}$ respectively. At adsorption-peak and desorption-peak vapor activities (i.e. $a=a_{\mathrm{ap}}$ and $a=a_{\mathrm{dp}}$, which are known experimentally), we denote: (i) the activity of the solution as $a_{\mathrm{sol}}\left(x_{\mathrm{ap}}\right)$ and $a_{\mathrm{sol}}\left(x_{\mathrm{dp}}\right)$, (ii) the corresponding dissolved mole fraction of solute as $x_{\mathrm{ap}}$ and $x_{\mathrm{dp}}$, and (iii) the surface tension of solution as $\gamma_{\mathrm{sol}}\left(x_{\mathrm{ap}}\right)$ and $\gamma_{\mathrm{sol}}\left(x_{\mathrm{dp}}\right)$. Upon substituting Equation 24 for the activity of solution and surface tension as a function of the dissolved mole fraction of solute, $x$, at vapor activities, $a_{\mathrm{ap}}$ and $a_{\mathrm{dp}}$, we solve for $x_{\mathrm{ap}}$ and $x_{\mathrm{dp}}$ using implicit relation in $x_{\mathrm{ap}}$ and $x_{\mathrm{dp}}$ :

$$
\begin{aligned}
& \left(\frac{a_{\mathrm{ap}}}{a_{\mathrm{sol}}\left(x_{\mathrm{ap}}\right)}\right)^{1 / \gamma_{\mathrm{sol}}\left(x_{\mathrm{ap}}\right)}=a_{\mathrm{w}, \mathrm{at}}^{1 / \gamma_{\mathrm{w}}} \\
& \left(\frac{a_{\mathrm{dp}}}{a_{\mathrm{sol}}\left(x_{\mathrm{dp}}\right)}\right)^{1 / \gamma_{\mathrm{sol}}\left(x_{\mathrm{dp}}\right)}=a_{\mathrm{w}, \mathrm{dt}}^{1 / \gamma_{\mathrm{w}}}
\end{aligned}
$$

where activity of the solution, $a_{\text {sol }}$, and surface tension, $\gamma_{\text {sol }}$, is known as empirical functions of dissolved mole fraction of solute, $x$ (See SI). Once we have evaluated $x_{\mathrm{ap}(\mathrm{dp})}$ from Equation 25 , we calculate the volume fraction of liquid filled pores, $\phi_{\mathrm{ap}(\mathrm{dp})}$, corresponding to vapor activity $a=a_{\mathrm{ap}(\mathrm{dp})}$, assuming that the total amount of solute remains constant during adsorption and desorption:

$$
\phi_{\mathrm{ap}(\mathrm{dp})}=\frac{V_{\mathrm{ap}(\mathrm{dp})}}{V_{\mathrm{s}}}=\frac{v_{\mathrm{sol}}\left(x_{\mathrm{ap}(\mathrm{dp})}\right) x_{\mathrm{s}}}{v_{\mathrm{sol}}\left(x_{\mathrm{s}}\right) x_{\mathrm{ap}(\mathrm{dp})}},
$$

where $v_{\mathrm{sol}}\left(x_{\mathrm{s}}\right)$ and $v_{\mathrm{sol}}\left(x_{\mathrm{ap}(\mathrm{dp})}\right)$ are the molar volume of the solutions corresponding to dissolved mole fraction of solute being $x_{\mathrm{s}}$ and $x_{\mathrm{ap}(\mathrm{dp})}$ respectively.

We note that in the case of a pure fluid filling the pore network, desorption occurs in a very narrow range of vapor activity, as illustrated in Figure 2. This is contrary to the 

volume fraction filled developed above. As a result, the calculations above cannot be applied to the case of pure water but only to the case of solutions.

\section{Results and Discussion}

\section{Qualitative features of reflectance Isotherms}

In this subsection, we discuss qualitatively each of the noted features in Figure 1(b) as a function of the activity of bulk $\mathrm{NaCl}$ (Figure 3) and $\mathrm{LiCl}$ (Figure 4) solutions, $a_{\mathrm{s}}$, within the pore volume. Figures 3 and 4 present reflectance isotherms for $\mathrm{NaCl}$ (Fig 3) and $\mathrm{LiCl}$ (Fig 4) solutions for a range of bulk-solution activities and corresponding mole fraction of solute, for $\mathrm{NaCl}: a_{\mathrm{s}}=0.794 \pm 0.020-0.947 \pm 0.005\left(\equiv x_{\mathrm{s}}: 0.0254-0.0948\right)$, and, for $\mathrm{LiCl}$ : $a_{\mathrm{s}}=0.260 \pm 0.016-0.942 \pm 0.005\left(\equiv x_{\mathrm{s}}: 0.0238-0.1980\right)$. In the case of $\mathrm{LiCl}$, we collected isotherms at additional, intermediate activities; we present these in Figure S11.

The reflectance isotherms for pure solvent (here, water, Figure 2) have features characteristic of mass isotherms: plateaus on both adsorption and desorption branches at higher activity ( $a>a_{\mathrm{w}, \text { at }}$ and $a>a_{\mathrm{w}, \mathrm{dt}}$ ), associated with entirely filled pores; reversible variation for lower vapor activity $(a<0.4)$, associated with molecules adsorbing on the pore walls; and hysteresis between adsorption and desorption branch in between these two reversible regimes. In addition, we observe, a feature characteristic only of the reflectance isotherms, where scattering increases, or, in other words, reflectance decreases before the end of filling and the onset of draining on adsorption $\left(a_{\mathrm{w}, \mathrm{ap}}\right)$ and desorption branch $\left(a_{\mathrm{w}, \mathrm{dp}}\right)$, respectively. This observation is consistent with the decrease in transmission observed on adsorption and desorption branch in porous Vycor. ${ }^{17,18}$

Qualitatively, the reflectance isotherms for $\mathrm{NaCl}$ and $\mathrm{LiCl}$ solutions are similar to those of the pure solvent, but differ in two aspects: 1) Trajectory of desorption curve: the desorption curve, for pure solvent, falls sharply onto adsorption curve, while, for the case of solution, 

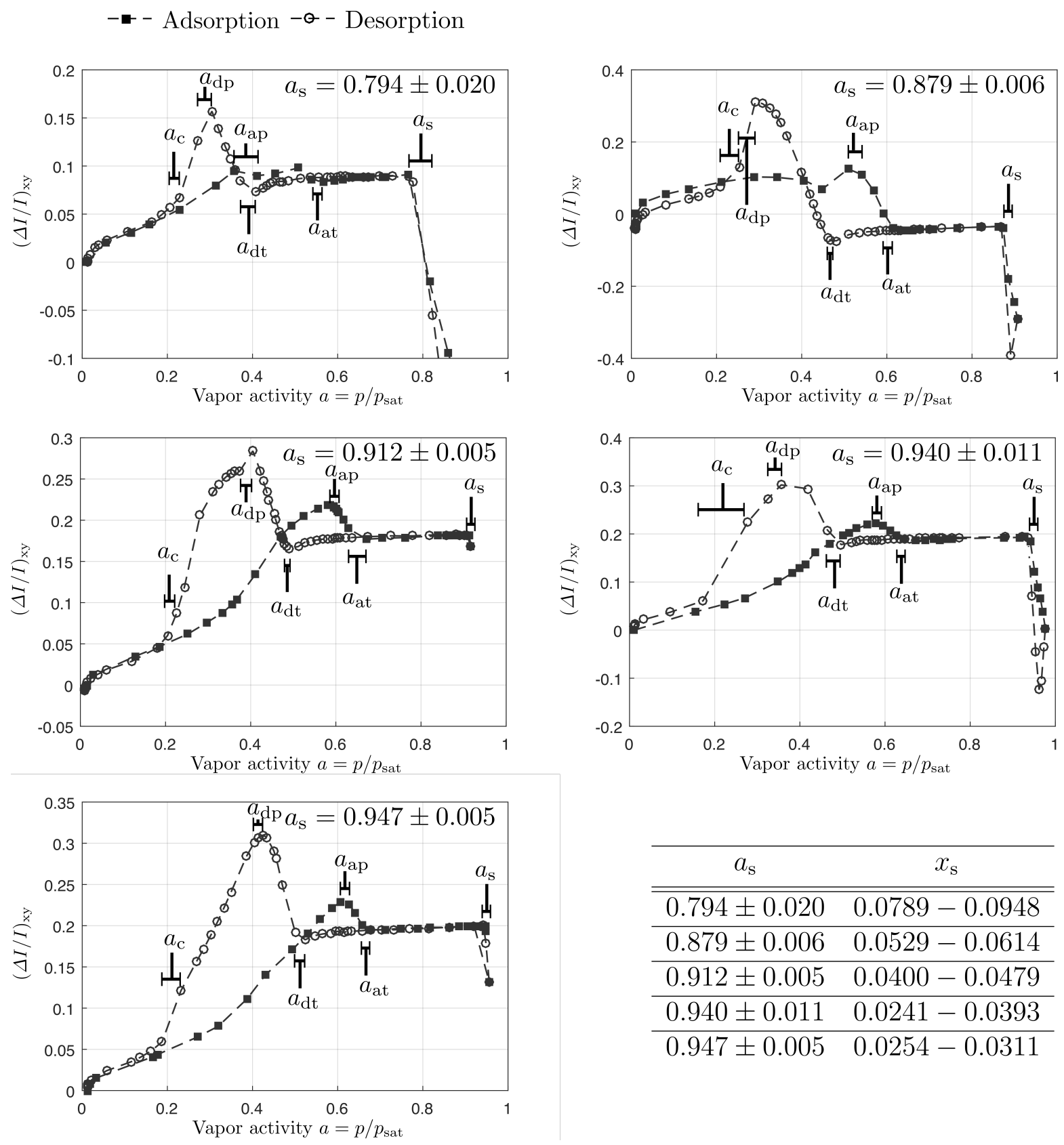

\begin{tabular}{cc}
\hline$a_{\mathrm{s}}$ & $x_{\mathrm{s}}$ \\
\hline \hline $0.794 \pm 0.020$ & $0.0789-0.0948$ \\
\hline $0.879 \pm 0.006$ & $0.0529-0.0614$ \\
\hline $0.912 \pm 0.005$ & $0.0400-0.0479$ \\
\hline $0.940 \pm 0.011$ & $0.0241-0.0393$ \\
\hline $0.947 \pm 0.005$ & $0.0254-0.0311$
\end{tabular}

Figure 3: Reflectance isotherms for $\mathrm{NaCl}$ solutions at various concentrations. Concentration is measured by the bulk-solution activity, $a_{\mathrm{s}}$, and corresponding mole fraction, $x_{\mathrm{s}}$, as indicated in legends. Closed squares and open circles represent adsorption branch and desorption branch respectively. Table lists all solutions studied. 

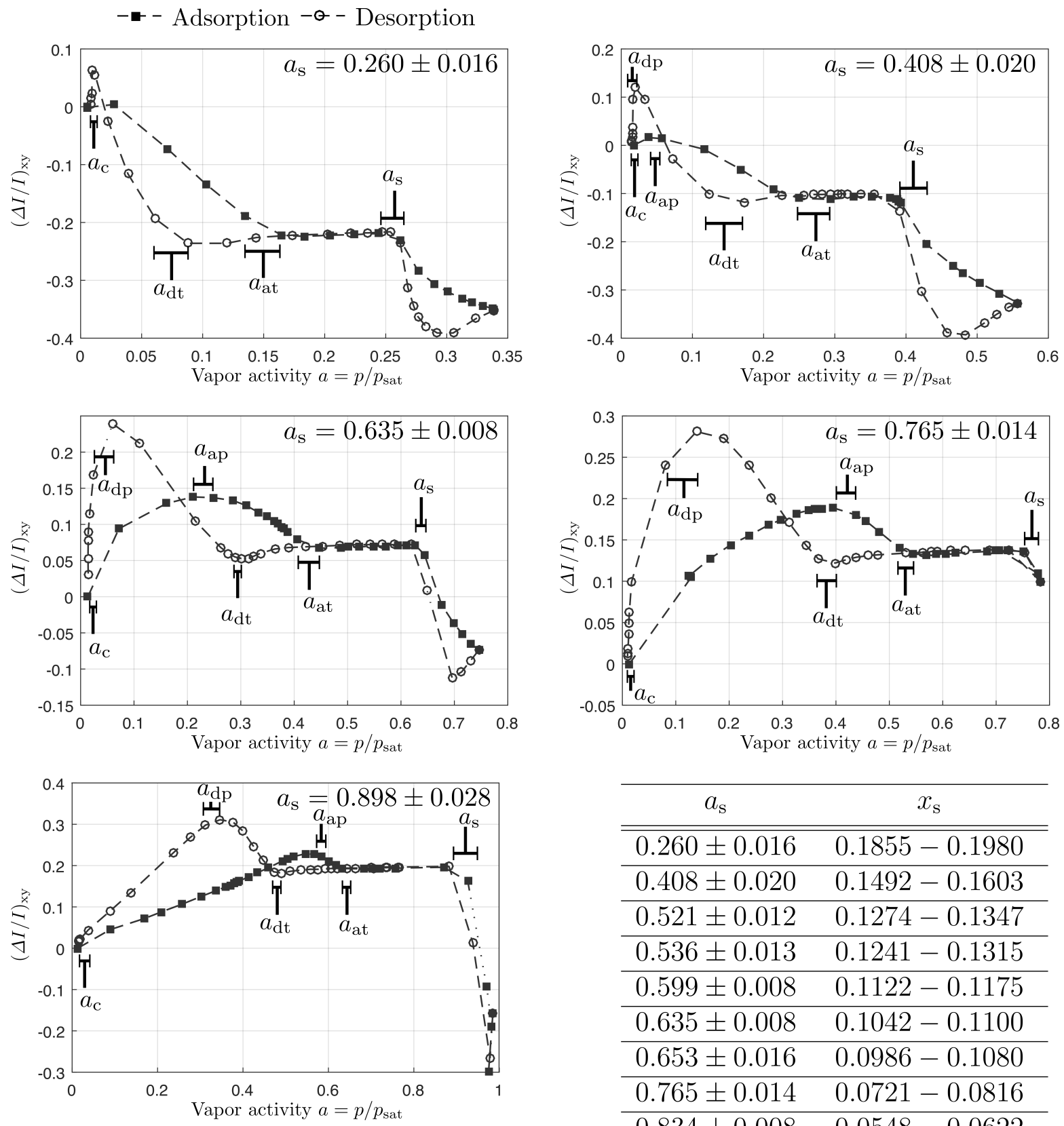

\begin{tabular}{cc}
\hline$a_{\mathrm{s}}$ & $x_{\mathrm{s}}$ \\
\hline \hline $0.260 \pm 0.016$ & $0.1855-0.1980$ \\
\hline $0.408 \pm 0.020$ & $0.1492-0.1603$ \\
\hline $0.521 \pm 0.012$ & $0.1274-0.1347$ \\
\hline $0.536 \pm 0.013$ & $0.1241-0.1315$ \\
\hline $0.599 \pm 0.008$ & $0.1122-0.1175$ \\
\hline $0.635 \pm 0.008$ & $0.1042-0.1100$ \\
\hline $0.653 \pm 0.016$ & $0.0986-0.1080$ \\
\hline $0.765 \pm 0.014$ & $0.0721-0.0816$ \\
\hline $0.834 \pm 0.008$ & $0.0548-0.0622$ \\
\hline $0.847 \pm 0.015$ & $0.0489-0.0604$ \\
\hline $0.898 \pm 0.028$ & $0.0306-0.0495$ \\
\hline $0.942 \pm 0.005$ & $0.0238-0.0304$
\end{tabular}

Figure 4: Examples of reflectance isotherms for $\mathrm{LiCl}$ solutions (see rest in SI, Figure S11) at various concentrations. Concentration is measured by the bulk-solution activity, $a_{\mathrm{s}}$, and corresponding mole fraction, $x_{\mathrm{s}}$, as indicated in legend. Closed squares and open circles represent adsorption branch and desorption branch respectively. Table lists all solutions studied. 
the desorption curve follows a more continuous and globally much wider trajectory before collapsing onto the adsorption curve (see Figure 2 and Figure 3, 4). Similarly, we observe sharp increase in scattering for the case of pure water for vapor activity for $a_{\mathrm{w}, \mathrm{dp}}<a<a_{\mathrm{w}, \mathrm{dt}}$, while, we observe continuous increase in scattering on desorption branch for $a_{\mathrm{dp}}<a<a_{\mathrm{dt}}$. These behaviors agree with our hypothesis that, unlike for the pure solvent, the recession of solution and the associated increase in concentration (equivalently, decrease in activity of solution) below $a_{\mathrm{dt}}$ allows for continuous re-establishment of equilibrium (Figure 1(f)). This phenomenon allows us to determine the filling fraction of the porous media (from corresponding solute concentration and assuming that moles of solute remain constant) at a given vapor activity (Equation 26). 2) Closure of hysteresis loop: For pure solvent, we observe the characteristic step down in desorption branch leading to closure of hysteresis loop (Figure 2), which is attributed to various effects such as cavitation induced emptying of pores, pore-blocking and percolation. ${ }^{15,17,40}$ In contrast, we observe gradual closure of hysteresis loop for the case of solutions. From isotherms in Figure 3 and 4, we note that the vapor activity associated with closure of hysteresis loop, $a_{\mathrm{c}}$, is independent of bulk-solution activity, $a_{\mathrm{s}}$, and occurs at fixed value of vapor activity, $a$; this observation indicates that the hysteresis loop collapses at a fixed value of solute concentration (solute concentration is only a function of vapor activity for vapor activity, $a$, lower than $a_{\mathrm{dt}}$ and independent of the initial activity of the solution $\left(a_{\mathrm{s}}\right)$, or, equivalently, the initial concentration of the solution $\left(x_{\mathrm{s}}\right)$, Equation 16, refer Theory), as should occur with crystallization (Equation 22, refer Theory). Later in this section, we explain this observation quantitatively.

We now turn to a quantitative analysis of the variation of features observed in the isotherms for solutions of $\mathrm{NaCl}$ (Figure 3) and $\mathrm{LiCl}$ (Figure 4). 
Figure 5: Effect of solute concentration on adsorption and desorption: Adsorption-tension activity, $a_{\mathrm{at}}$ (completion of pore filling), and desorption-tension activity, $a_{\mathrm{dt}}$ (onset of pore emptying) from isotherms as in Figure 3 and 4 . Vapor activities for solution as predicted from Kelvin equation (Equation 18) are shown in dashed lines for $\mathrm{LiCl}$ and $\mathrm{NaCl}$ solutions. Experimental values of $a_{\text {at }}$ and $a_{\mathrm{dt}}$ from isotherms as in Figures 3 and 4 are shown for $\mathrm{LiCl}$ as solid circles $(\bullet, \bullet)$ and for $\mathrm{NaCl}$ as translucent diamonds $(\diamond, \diamond)$. Adsorption-tension activity and desorption-tension activity for pure water, i.e., $a_{\mathrm{w}, \text { at }}$ and $a_{\mathrm{w}, \mathrm{dt}}$, as extracted from Figure 2, are plotted as solid circles $(\circ, \circ)$. Error bars for $a_{\mathrm{s}}, a_{\mathrm{at}}$ and $a_{\mathrm{dt}}$ represent the uncertainty in experimental values extracted from the isotherm (see Methods). In Equation 18, surface tension of solution as function of $\mathrm{NaCl}$ and $\mathrm{LiCl}$ concentration is reported in SI (Figure S1) and surface tension for pure water is taken to be $73.5 \mathrm{mN} / \mathrm{m}$ at $15^{\circ} \mathrm{C}$.

\section{Shift in adsorption-tension activity $\left(a_{\text {at }}\right)$ and desorption-tension ac- tivity $\left(a_{\mathrm{dt}}\right)$ as a function of solute concentration}

Figure 5 presents the activities, $a_{\mathrm{at}}$ and $a_{\mathrm{dt}}$, extracted from the isotherms in Figures 3 and Figure 4 as functions of $a_{\mathrm{s}}$. In the same figure, we plot the predictions of the Kelvin equation for solutions with pure water as reference based on Equation S12. The theoretical prediction matches well with the experimental values of $a_{\text {at }}$ and $a_{\mathrm{dt}}$ for both $\mathrm{LiCl}$ (solid filled circles) and $\mathrm{NaCl}$ (diamonds) solutions with pure water (transparent filled circles; $a_{\mathrm{s}}=1$ ) as reference point. This agreement supports the hypothesized state of solution in the pore at the vapor 
activity, $a_{\mathrm{at}}$ and $a_{\mathrm{dt}}$, i.e., the solution is under characteristic maximum tension (negative pressure) before the end of filling and the onset of draining on adsorption and desorption branch respectively.

The agreement shown above between theoretical and experimental predictions of $a_{\text {at }}$ and $a_{\mathrm{dt}}$ along with the hypothesized state of solution under tension at vapor activity $a_{\text {at }}$ and $a_{\mathrm{dt}}$ (Figure $1(\mathrm{e}, \mathrm{i})$ ) and the hypothesized state of no-tension condition at vapor activity, $a_{\mathrm{s}}$ (Figure 1(c)), indicates that the same theoretical principles explain the state of solution in the pores on adsorption plateau $\left(a_{\mathrm{at}}<a<a_{\mathrm{s}}\right)$ and desorption plateau $\left(a_{\mathrm{dt}}<a<a_{\mathrm{s}}\right)$ (Figure $1(\mathrm{~d}, \mathrm{j})$ ). In other words, the tension in the pores at a given activity in both the adsorption plateau $\left(a_{\mathrm{at}}<a<a_{\mathrm{s}}\right)$ and the desorption plateau $\left(a_{\mathrm{dt}}<a<a_{\mathrm{s}}\right)$ should be given by the modified Kelvin equation (Equation 11) and the pressure difference should be balanced mechanically with varying contact angle between liquid meniscus and the pore wall as given by Laplace equation (Equation 12, Figure 1(d, j)). As suggested by Equation 11, salt concentration (activity of solution or osmotic pressure) tunes the tension in nanopores for any given vapor activity. The success of our predictions also supports our assumption that bulk thermodynamic properties $\left(\gamma_{\text {sol }}, a_{\text {sol }}\right)$ remain relevant in the pores studied here with diameter of $\sim 3 \mathrm{~nm}$.

We also note that when increasing the vapor activity above $a_{\mathrm{c}}$, the crystal present in the pore needs to transition into a solution (a process known as deliquescence). Since, we know that at the upper closure of the adsorption branch (vapor activity $a_{\text {at }}$ ) the pores are filled with solution, it follows that $a_{\text {at }}$ provides an upper bound on the deliquescence activity. Interestingly, for $\mathrm{NaCl}$ solutions, the experimental values of $a_{\text {at }}$ is well below the bulk deliquescence point of $\mathrm{NaCl}$ crystals at $a \sim 0.75,{ }^{41}$ indicating strong confinement effects on the deliquescence point of crystals in nanopores. 

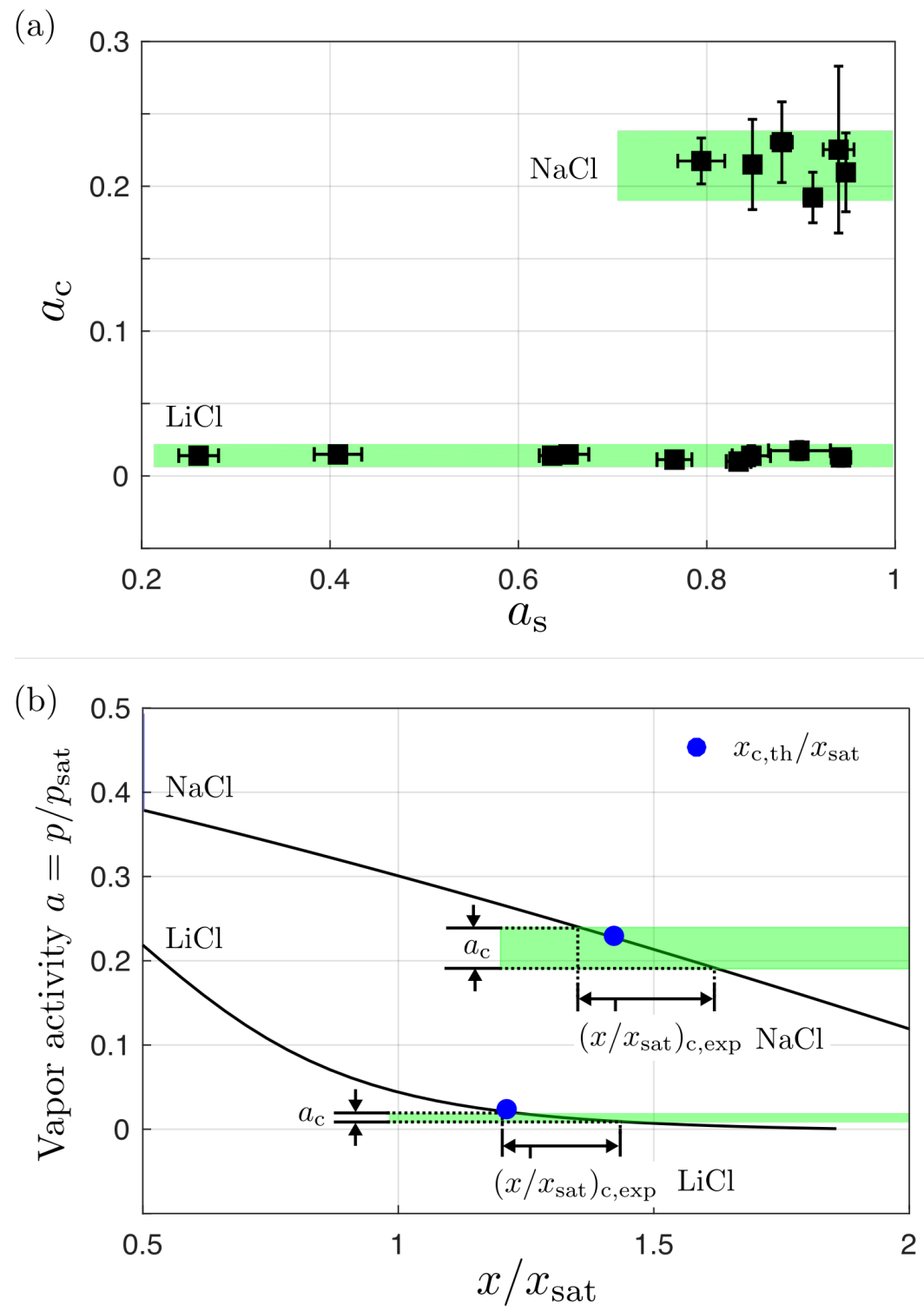

Figure 6: Effect of initial solute concentration on the lower closure point of the hysteresis cycles due to crystallization $\left(a_{\mathrm{c}}\right)$, and corresponding supersaturations. (a) Experimental values of crystallization activity $\left(a_{\mathrm{c}}\right)$ as a function of bulk-solution activity $\left(a_{\mathrm{s}}\right)$ for $\mathrm{NaCl}$ from isotherms as in Figure 3 and for $\mathrm{LiCl}$ as in isotherms from Figure 4, where $a_{\mathrm{c}}$ is the lower point of closure of the adsorption and desorption branches. The shaded green region is the range given by the average of all error bars on values of $a_{\mathrm{c}}$ around the average values of $a_{\mathrm{c}}$. (b) The theoretical and experimental supersaturation for crystallization of $\mathrm{LiCl}$ and $\mathrm{NaCl}$ solutions. The solid black line are the equilibrium vapor activity vs supersaturation for $r_{\mathrm{p}}=1.4 \mathrm{~nm}$ for $\mathrm{NaCl}$ and $\mathrm{LiCl}$ solutions as indicated. The blue dots are the values of theoretical supersaturation for the onset of crystallization, $x_{\mathrm{c}, \mathrm{th}} / x_{\mathrm{sat}}$, found from the classical nucleation theory for $r_{\mathrm{c}}=r_{\mathrm{p}}$ (Equation 17, 18) and correspond to $r_{\mathrm{p}}=1.4 \mathrm{~nm}$. The shaded green is the range of averaged experimental vapor activity, $a_{\mathrm{c}}$ (same as in $\left.6(\mathrm{a})\right)$. $\left(x / x_{\mathrm{sat}}\right)_{\mathrm{c}, \exp }$ is the supersaturation associated with $a_{\mathrm{c}}$ (obtained experimentally) for onset of crystallization. 


\section{Crystallization within pores at large supersaturations}

As we have noted above, the results presented in Figures 3 and 4 show that the lower closure of the hysteresis cycle of the reflectance isotherms (vapor activity, $a_{\mathrm{c}}$ ) always occurs at similar vapor activities for a given solute, regardless of the initial concentration of that solute in the porous network. In other words, $a_{\mathrm{c}}$ is independent of the bulk-solution activity $a_{\mathrm{s}}$ : in the case of $\mathrm{NaCl}$, we have $a_{\mathrm{c}}=0.215 \pm 0.025$ for all measured isotherms (with $a_{\mathrm{s}}$ varying from 0.79 and 0.95 ), while for $\mathrm{LiCl}, a_{\mathrm{c}}=0.014 \pm 0.009$ (with $a_{\mathrm{s}}$ varying from 0.26 to 0.94 ), see Figure 6a. We note that while closure for $\mathrm{LiCl}$ is consistent, it happens at very low vapor pressures that are at the limit of values that we can resolve with our experimental system.

As explained in the Theory section, when the pores are not full (i.e. during desorption below $a_{\mathrm{dt}}$ or adsorption below $a_{\mathrm{at}}$ ), there is a direct relation between the imposed vapor activity, $a$, and the solute concentration in the pores (Equation 16); thus, the observation of at constant $a_{\mathrm{c}}$ suggests a process that occurs at a fixed solute concentration. In Figure $6 \mathrm{~b}$, we plot this relation for both $\mathrm{NaCl}$ and $\mathrm{LiCl}$ solutions (black lines), with concentration expressed in terms of the supersaturation ratio $x / x_{\mathrm{sat}}$, where $x_{\mathrm{sat}}$ is the mole fraction of solute in saturated solution at $T=15^{\circ} \mathrm{C}$ (for $\mathrm{NaCl}$ : $x_{\text {sat }}=0.099$, for LiCl: $x_{\text {sat }}=0.242$ ). These curves allow us to translate the measured values of $a_{\mathrm{c}}$ (Figure 6a) into corresponding supersaturations, and we obtain $x / x_{\text {sat }}=1.48 \pm 0.13$ for $\mathrm{NaCl}$ and $x / x_{\text {sat }}=1.34 \pm 0.13$ for LiCl (Figure 6b).

These values are consistent with predictions from CNT, assuming sterically hindered homogeneous nucleation of the salt crystal (see Theory): on numerically solving Equation 22 for $r_{\mathrm{c}}=r_{p}$, we obtain the theoretical value for dissolved mole fraction, $x_{\mathrm{c}, \mathrm{th}}$, that should be sufficient for crystallization; we find: $x_{\mathrm{c}, \mathrm{th}}=0.140$ for $\mathrm{NaCl}$ and $x_{\mathrm{c}, \text { th }}=0.285$ for $\mathrm{LiCl}$ for $r_{\mathrm{p}}=1.4 \mathrm{~nm}$, corresponding to supersaturations of 1.408 and 1.176, and vapor activities of 0.230 and 0.022 , respectively (blue dots in Figure 6b). These values match well with the experimental values of vapor activities $a_{\mathrm{c}}$ (shaded green region, Figure 6(b)), suggesting that the closure of the hysteresis loops at $a_{\mathrm{c}}$ is associated with the crystallization of the salt 
We note that the values of supersaturations extracted from the experimental data and theoretically predicted by CNT are sensitive to the choice of pore size and wettability; in the SI, we propagate typical uncertainties on pore radius and contact angle to evaluate their impact (see Figures S9 and S10). The leading effect is that of pore size; for example, assuming $r_{\mathrm{p}}=1.4 \pm 0.2 \mathrm{~nm}$ yields uncertainties for $x / x_{\text {sat }}$ in the range $1.41 \pm 0.32$ and $1.34 \pm 0.17$ for experimental supersaturations of $\mathrm{NaCl}$ and $\mathrm{LiCl}$, respectively, and $1.42 \pm 0.07$ and $1.18 \pm 0.03$ for theoretical supersaturations of $\mathrm{NaCl}$ and $\mathrm{LiCl}$, respectively (see $\mathrm{SI}$ ).

An interesting aspect in $\mathrm{CNT}$ is that the second term inside the exponential of the right hand side of Equation 22 incorporates the effect of negative pressure (tension) in inducing crystallization. Tension in the liquid is related to the large capillary pressure of the curved nano-menisci at the solution-vapor interface (see Theory). Including this term reduces theoretical supersaturations for crystallization by $\sim 6 \%$ for $\mathrm{NaCl}$ and $\sim 1 \%$ for $\mathrm{LiCl}$. Given our experimental uncertainties, it is however difficult to isolate this effect in our data.

Finally, we note that the supersaturations estimated here for $\mathrm{NaCl}$ are comparable to recent measurements of crystallization in capillary tubes $\left(x / x_{\text {sat }} \simeq 1.6\right.$ according to several authors ${ }^{42-44}$ ), where crystal nucleation is expected to be limited by kinetics and not steric confinement as assumed in our CNT model. Other supersaturation estimates range from $x / x_{\text {sat }} \approx 1.05-1.1$ in porous media with large pore sizes, ${ }^{45-47}$ to $x / x_{\text {sat }} \simeq 2.3-2.6$ in levitated droplets. ${ }^{48,49}$ Further studies are required to identify the relative effects of confinement, heterogeneous nucleation and kinetics to explain this variability of observed supersaturations.

\section{Percolation-induced optical scattering}

As discussed in Theory section, we interpret the maxima in reflectance on the adsorption and desorption isotherms as arising due to heterogeneities in the distribution of the liquid and vapor phases within the pores. We observe continuous, unimodal change in reflectance on both adsorption and desorption branches of Figure 3-4. As discussed in the Theory section, 
(a)

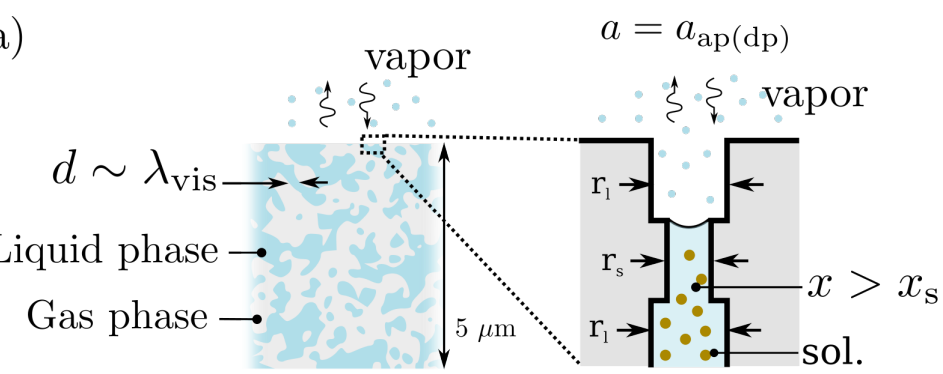

(b)
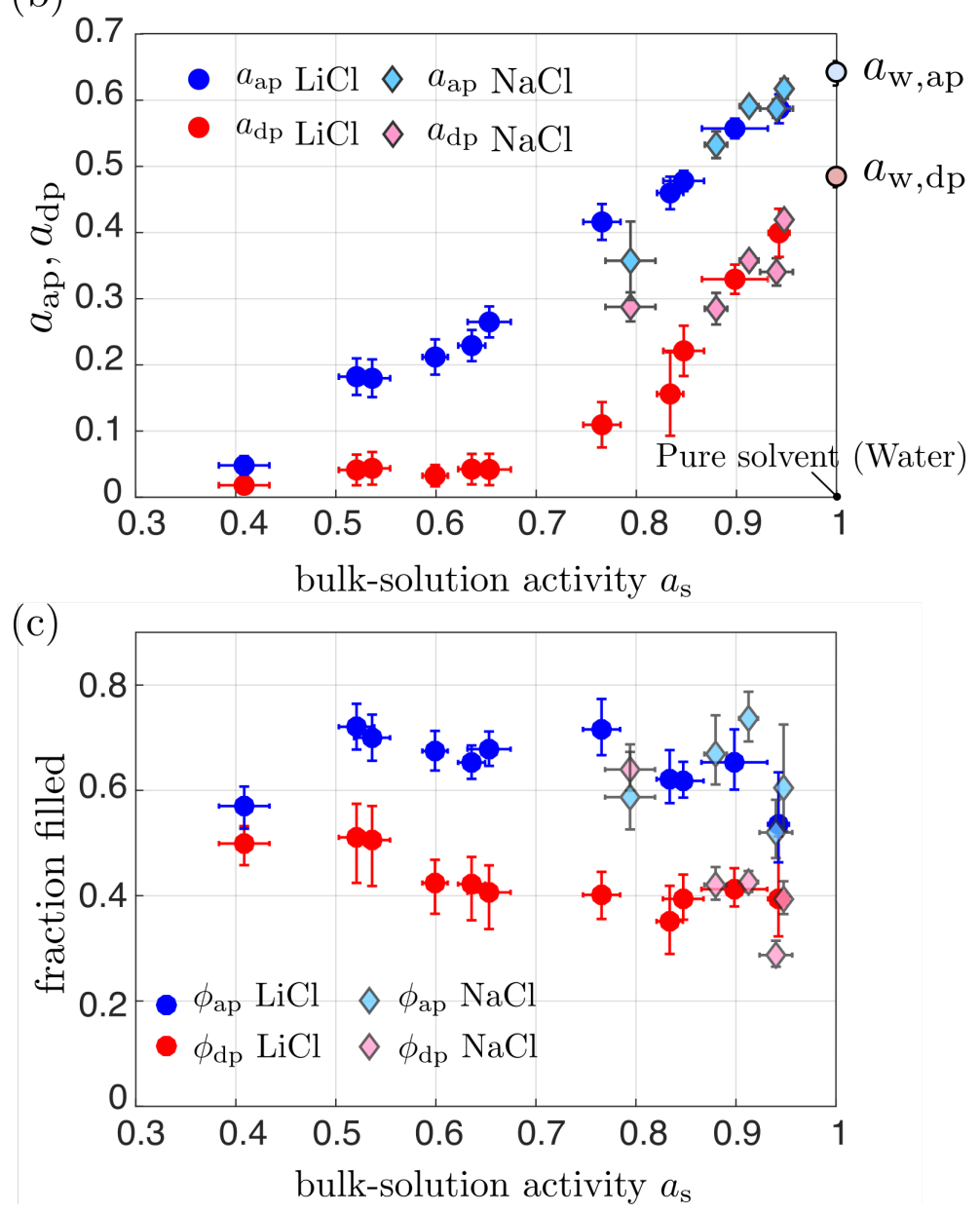

Figure 7: Enhanced scattering as a result of the distribution of solution at adsorption-peak $\left(a_{\mathrm{ap}}\right)$ and desorption-peak $\left(a_{\mathrm{dp}}\right)$ vapor activity. (a) Schematic diagrams of the hypothetical state of pore solution at vapor activity, $a=a_{\mathrm{ap}(\mathrm{dp})}$, where pore space connectivity and pore size non-uniformity lead to clusters of liquid and gas phase with cluster dimension, $d$, of order of the visible wavelength of light, $\lambda_{\text {vis }}$. Concentration of the pore solution increases as meniscus recedes with decreasing vapor activity $\left(x>x_{\mathrm{s}}\right)$; larger pores connected to smaller pores $\left(r_{\mathrm{s}}<r_{1}\right)$ remain undrained due to pore-blocking effect (See Theory Section). (b) Experimental values of adsorption-peak activity $\left(a_{\mathrm{ap}}\right)$ and desorption-peak activity $\left(a_{\mathrm{dp}}\right)$ as a function of bulk-solution activity $\left(a_{\mathrm{s}}\right)$ for $\mathrm{NaCl}$ solution and $\mathrm{LiCl}$ solution. (c) Calculated fraction of pore volume filled (based on Equation 26), $\phi_{\text {ap }}$ and $\phi_{\mathrm{dp}}$, at vapor activity, $a_{\mathrm{ap}}$ and $a_{\mathrm{dp}}$, for $\mathrm{LiCl}$ and $\mathrm{NaCl}$ solutions respectively. Error bars for $a_{\mathrm{s}}, a_{\mathrm{ap}}$ and $a_{\mathrm{dp}}$ represent the uncertainty in experimental values extracted from the isotherms. 
we speculate that disordered fluid networks of size comparable to the wavelength of light are formed on both filling and draining of solution-filled porous medium during respectively adsorption and desorption, and that this results in enhanced scattering, as observed in our isotherms. Figure $7(\mathrm{a})$ presents the hypothetical schematic diagram of filling of the porous network at vapor activities corresponding to maximum scattering on adsorption $\left(a_{\text {ap }}\right)$ and desorption $\left(a_{\mathrm{dp}}\right)$. As discussed earlier in the theory section, formation of clusters of liquid and vapor associated with the increase in light scattering results from two underlying causes: the pore size distribution (pore blocking percolation effect) and continuous change of the activity, or, equivalently, of the concentration of the solution (Equation 26, Figure 7(a)).

We observe that adsorption-peak activity, $a_{\mathrm{ap}}$, and desorption-peak activity, $a_{\mathrm{dp}}$, as a function of $a_{\mathrm{s}}$ (Figure 7 (b)) show similar trend for $\mathrm{NaCl}$ and $\mathrm{LiCl}$ solutions. On the adsorption branch, for vapor activity below $a_{\text {at }}$, a confined solution can be in equilibrium with vapor of higher activity by decreasing its concentration by filling additional pore volume. Similarly, during desorption, a confined solution can be in equilibrium with the vapor by recession of the pore solution, in other words, by increasing the concentration.

We used $x_{\mathrm{ap}(\mathrm{dp})}$ calculated from Equation 25 in Equation 26 to calculate the fraction of pore volume filled, $\phi_{\mathrm{ap}(\mathrm{dp})}$ (Figure $7(\mathrm{c})$ ), at the vapor activity of maximum scattering, $a=a_{\mathrm{ap}(\mathrm{dp})}$. Reflectance peaks occur in two distinct ranges of volume fraction of filled pores. Upon adsorption, the filling fraction, $\phi_{\mathrm{ap}}$, corresponding to vapor activity, $a_{\mathrm{ap}}$, occurs from $0.53-0.69$ of total pore volume; upon desorption, the filling fraction, $\phi_{\mathrm{dp}}$, corresponding to vapor activity, $a_{\mathrm{dp}}$, occurs from $0.35-0.52$. Note that these values are the same for both $\mathrm{NaCl}$ and $\mathrm{LiCl}$ solutions. These results indicate that there is relatively narrow variation in $\phi_{\mathrm{ap}}$ and $\phi_{\mathrm{dp}}$ for solutions with wide range of bulk-solution activity, $a_{\mathrm{s}}$, and there are no significant trends in $\phi_{\mathrm{ap}}$ and $\phi_{\mathrm{dp}}$ as function of $a_{\mathrm{s}}$. Since the filing fraction is not an explicit function of pore radius, evaluating the effect of pore size distribution on filling fraction here is as such non-trivial and requires detailed studies of pore-scale processes in disordered pore networks. The theoretical filling fraction associated with the critical percolation threshold for 
square and triangular lattice ranges from 0.3 to $0.6 .{ }^{50}$ Therefore, the constant filing fractions obtained experimentally at $a_{\mathrm{ap}}$ and $a_{\mathrm{dp}}$ hint at percolation induced scattering. The fact that these values are also independent of solute suggests that increased scattering is a result of physical formations, and not influenced by the chemical composition of these clusters.

\section{Conclusion}

In conclusion, we have demonstrated the use of reflectance isotherms to evaluate the thermodynamic state of solutions in equilibrium with subsaturated vapor in mesoporous silicon. This technique could be generalized to other mesoporous materials. We have shown that a modified Kelvin-Laplace equation for solution quantitatively explains the features in the isotherms for pore liquid of various compositions. We have found that collapse of hysteresis loop is independent of activity of bulk solution and matches well with the theoretical prediction for onset of crystallization derived from Classical Nucleation Theory for suppression of crystallization due to steric hindrance of critical nucleus by the pores. However, we are uncertain about the mode of nucleation and the role of kinetics in crystallization. Our results also suggest strongly shifted deliquescence of confined $\mathrm{NaCl}$ crystals compared to the bulk. Our results show the existence of minima of reflectance upon both adsorption and desorption, which are consistent with invasion percolation resulting in pore-space correlation and pore-blocking-induced scattering. The successful use of bulk properties and thermodynamic arguments to explain these results imply that they remain relevant to strongly confined scenarios $(\sim 3 \mathrm{~nm})$.

Reflectance isotherms of mesoporous materials contribute to our fundamental understanding of wetting and drying with nano-confined solutions. They provide a convenient way to investigate the impact of solutes on sorption dynamics and thermodynamics and estimate the associated stresses generated in the medium. This understanding could improve our comprehension of phenomena of natural and industrial interest such as damage or crack- 
ing, and aid the development of technologies involving confined solutions for desalination, water purification and geological saline buffers.

\section{Acknowledgement}

This work was supported by the National Science Foundation (IIP-1500261), the Air Force Office of Scientific Research (FA9550-15-1-0052), the U.S. Department of Agriculture (201567021-22844), and was performed in part at the Cornell NanoScale Facility, a member of the National Nanotechnology Infrastructure Network (National Science Foundation; Grant No. ECCS-1542081).

\section{Supporting Information Available}

Supporting Information1.pdf:

- Properties of $\mathrm{NaCl}$ and $\mathrm{LiCl}$ solution as a function of concentration

- Derivation of thermodynamic condition for crystallization

- Additional Reflectance Isotherms

\section{References}

(1) Scherer, G. W. Crystallization in pores. Cement and Concrete Research 1999, 29, $1347-1358$.

(2) Flatt, R. J. Salt damage in porous materials: How high supersaturations are generated. Journal of Crystal Growth 2002, 242, 435-454.

(3) Berkowitz, B. Characterizing flow and transport in factures geological media: A review. Advances in Water Resources 1999, 25, 861-884. 
(4) Saadatpoor, E.; Bryant, S. L.; Sepehrnoori, K. New trapping mechanism in carbon sequestration. Transport in Porous Media 2010, 82, 3-17.

(5) Humplik, T.; Lee, J.; O’Hern, S. C.; Fellman, B. A.; Baig, M. A.; Hassan, S. F.; Atieh, M. A.; Rahman, F.; Laoui, T.; Karnik, R.; Wang, E. N. Nanostructured materials for water desalination. Nanotechnology 2011, 22, 292001.

(6) Selwitz, C.; Doehne, E. The evaluation of crystallization modifiers for controlling salt damage to limestone. Journal of Cultural Heritage 2002, 3, 205-216.

(7) Ruiz-Agudo, E.; Mees, F.; Jacobs, P.; Rodriguez-Navarro, C. The role of saline solution properties on porous limestone salt weathering by magnesium and sodium sulfates. Environmental Geology 2007, 52, 269-276.

(8) McCarthy, K. A.; Johnson, R. L. Transport of volatile organic compounds across the capillary fringe. Water Resources Research 1993, 29, 1675-1683.

(9) Silliman, S. E.; Berkowitz, B.; Simunek, J.; Van Genuchten, M. T. Fluid flow and solute migration within the capillary fringe. Ground Water 2002, 40, 76-84.

(10) Sghaier, N.; Prat, M.; Nasrallah, S. B. On ions transport during drying in a porous medium. Transport in Porous Media 2007, 67, 243-274.

(11) Huinink, H. P.; Pel, L.; Michels, M. A. J. How ions distribute in a drying porous medium: A simple model. Physics of Fluids 2002, 14, 1389-1395.

(12) Schoch, R. B.; Han, J.; Renaud, P. Transport phenomena in nanofluidics. Reviews of Modern Physics 2008, 80, 839-883.

(13) Pel, L.; Huinink, H.; Kopinga, K. Ion transport and crystallization in inorganic building materials as studied by nuclear magnetic resonance. Applied Physics Letters 2002, 81, $2893-2895$. 
(14) Rijniers, L. A.; Huinink, H. P.; Pel, L.; Kopinga, K. Experimental evidence of crystallization pressure inside porous media. Physical Review Letters 2005, 94, 23-26.

(15) Thommes, M. Physical adsorption characterization of nanoporous materials. ChemieIngenieur-Technik 2010, 82, 1059-1073.

(16) Valiullin, R.; Naumov, S.; Galvosas, P.; Kärger, J.; Woo, H.-J.; Porcheron, F.; Monson, P. A. Exploration of molecular dynamics during transient sorption of fluids in mesoporous materials. Nature 2006, 443, 965-968.

(17) Page, J. H.; Liu, J.; Abeles, B.; Deckman, H. W.; Weitz, D. A. Pore-space correlations in capillary condensation in Vycor. Physical Review Letters 1993, 71, 1216-1219.

(18) Soprunyuk, V.; Wallacher, D.; Huber, P.; Knorr, K.; Kityk, A. Freezing and melting of Ar in mesopores studied by optical transmission. Physical Review B 2003, 67, 1-7.

(19) Evans, R. Fluids adsorbed in narrow pores: Phase equilibria and structure. Journal of Physics: Condensed Matter 1999, 2, 8989-9007.

(20) Vincent, O.; Marguet, B.; Stroock, A. D. Imbibition Triggered by Capillary Condensation in Nanopores. Langmuir 2017, 33, 1655-1661.

(21) Vincent, O.; Szenicer, A.; Stroock, A. D. Capillarity-driven flows at the continuum limit. Soft Matter 2016, 12, 6656-6661.

(22) Vitagliano, V.; Lyons, P. A. Diffusion Coefficients for Aqueous Solutions of Sodium Chloride and Barium Chloride. Journal of the American Chemical Society 1956, 78, $1549-1552$.

(23) Sahimi, M. Flow and Transport in Porous Media and Fractured Rock; 2011; p 718.

(24) Huber, P. Soft matter in hard confinement: Phase transition thermodynamics, structure, texture, diffusion and flow in nanoporous media. Journal of Physics Condensed Matter 2015, 27. 
(25) Bocquet, L.; Charlaix, E. Nanofluidics, from bulk to interfaces. Chemical Society Reviews 2010, 39, 1073-1095.

(26) Wagner, W.; Pruss, A. International Equations for the Saturation Properties of Ordinary Water Substance. Revised According to the International Temperature Scale of 1990. Addendum to J. Phys. Chem. Ref. Data 16, 893 (1987). Journal of Physical and Chemical Reference Data 1993, 22, 783-787.

(27) Vincent, O.; Zhang, J.; Choi, E.; Zhu, S.; Stroock, A. D. How Solutes Modify the Thermodynamics and Dynamics of Filling and Emptying in Extreme Ink-Bottle Pores. Langmuir Just accepted Manuscript, 2019, 0, 1-46, PMID: 30681860.

(28) Conde, M. R. Properties of aqueous solutions of lithium and calcium chlorides: Formulations for use in air conditioning equipment design. International Journal of Thermal Sciences 2004, 43, 367-382.

(29) Olynyk, P.; Gordon, A. R. The Vapor Pressure of Aqueous Solutions of Sodium Chloride at 20, 25 and $30^{\circ}$ for Concentrations from 2 Molal to Saturation. Journal of the American Chemical Society 1943, 65, 224-226.

(30) Fukuta, N. Activation of Atmospheric Particles as Ice Nuclei in Cold and Dry Air. Journal of the Atmospheric Sciences 1966, 23, 741-750.

(31) Espinosa, J. R.; Vega, C.; Valeriani, C.; Sanz, E. The crystal-fluid interfacial free energy and nucleation rate of $\mathrm{NaCl}$ from different simulation methods. Journal of Chemical Physics 2015, 142 .

(32) Lanaro, G. Molecular Simulation of Nucleation and Dissolution of Alkali Halides. Ph.D. thesis, 2017.

(33) Volmer, M.; Weber, A. Keimbildung in übersättigten Gebilden. Z.Physik.Chem. 1926, $119,277$. 

Dämpfen. Annalen der Physik 1935, 416, 719-752. ena. The Journal of Chemical Physics 1939, 7, 538-547. ing and poromechanical modeling. 2012, 248. in the literature. Geological Survey Bulletin 1977, 5, C1-C36. Reference Data 1972, 1, 1047-1100. crystals. Journal of Crystal Growth 2005, 282, 455-469. 293. Letters 2014, 5, 890-895.

(35) Frenkel, J. A General Theory of Heterophase Fluctuations and Pretransition Phenom-

(36) Derluyn, H. Salt transport and crystallization in porous limestone: neutron-X-ray imag-

(37) Potter, R.; Brown, D. L. The volumetric properties of aqueous sodium chloride solution from $0^{\circ}$ to $500^{\circ} \mathrm{C}$ at pressures up to 2000 bars based on a regression of available data

(38) Hamer, W. J.; Wu, Y. Osmotic Coefficients and Mean Activity Coefficients of Uniunivalent Electrolytes in Water at 25 degrees Celsius. Journal of Physical and Chemical

(39) Steiger, M. Crystal growth in porous materials - I: The crystallization pressure of large

(40) Liu, H.; Zhang, L.; Seaton, N. A. Analysis of sorption hysteresis in mesoporous solids using a pore network model. Journal of Colloid And Interface Science 1993, 156, 285-

(41) Robinson, R. A.; Stokes, R. H. Electrolyte Soutions; Dover Publications, 1959.

(42) Desarnaud, J.; Derluyn, H.; Carmeliet, J.; Bonn, D.; Shahidzadeh, N. Metastability limit for the nucleation of $\mathrm{NaCl}$ crystals in confinement. Journal of Physical Chemistry

(43) Naillon, A.; Duru, P.; Marcoux, M.; Prat, M. Evaporation with sodium chloride crystallization in a capillary tube. Journal of Crystal Growth 2015, 422, 52-61. 
(44) Gupta, S.; Pel, L.; Steiger, M.; Kopinga, K. The effect of ferrocyanide ions on sodium chloride crystallization in salt mixtures. Journal of Crystal Growth 2015, 410, 7-13.

(45) Veran-Tissoires, S.; Prat, M. Evaporation of a sodium chloride solution from a saturated porous medium with efflorescence formation. Journal of Fluid Mechanics 2014, 749 , $701-749$.

(46) Noiriel, C.; Renard, F.; Doan, M. L.; Gratier, J. P. Intense fracturing and fracture sealing induced by mineral growth in porous rocks. Chemical Geology 2010, 269, 197209.

(47) Chatterji, S. A discussion of the paper "Crystallisation in pores"i£i by G.W. Scherer. Cement and Concrete Research 2000, 30, 669-671.

(48) Na, H. S.; Arnold, S.; Myerson, A. S. Cluster formation in highly supersaturated solution droplets. Journal of Crystal Growth 1994, 139, 104-112.

(49) Hargreaves, G.; Kwamena, N. O.; Zhang, Y. H.; Butler, J. R.; Rushworth, S.; Clegg, S. L.; Reid, J. P. Measurements of the equilibrium size of supersaturated aqueous sodium chloride droplets at low relative humidity using aerosol optical tweezers and an electrodynamic balance. Journal of Physical Chemistry A 2010, 114, 1806-1815.

(50) Stauffer, D.; Aharony, A. Introduction to Percolation Theory; 1991; Vol. 1; p 192. 


\section{Graphical TOC Entry}

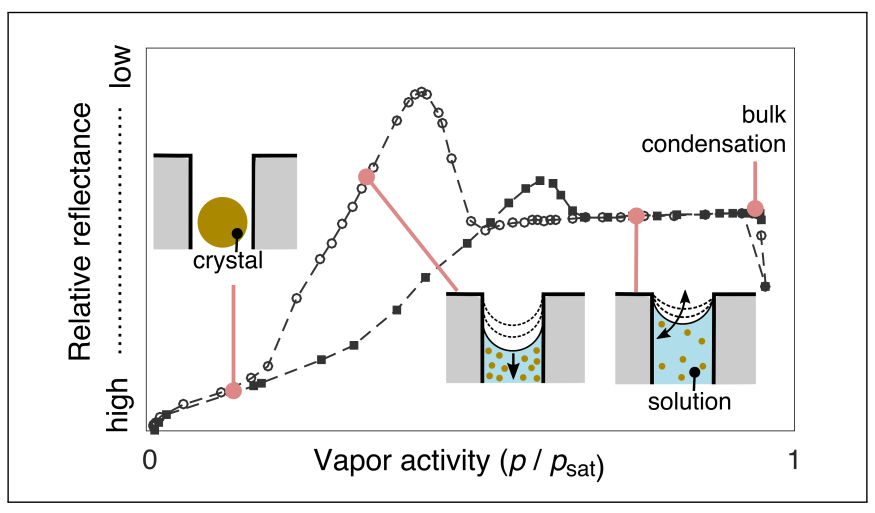

